\title{
Two new genera and three new subterranean species of Hydrobiidae (Caenogastropoda: Truncatelloidea) from Tunisia
}

\author{
Noureddine KHALLOUFI ${ }^{1}$, Mustapha BÉJAOUI ${ }^{2}$ \& Diana DELICADO ${ }^{3, *}$ \\ ${ }^{1,2}$ University of Carthage - Faculty of Sciences of Bizerte, LR01ES14, \\ Laboratory of Environment Biomonitoring, 7021 Jarzouna, Bizerte, Tunisia. \\ ${ }^{3}$ Justus Liebig University Giessen, Department of Animal Ecology \& Systematics, \\ Heinrich-Buff-Ring 26-32 IFZ, 35392 Giessen, Germany. \\ *Corresponding author: didelicado@gmail.com \\ 1Email: khalnour2003@yahoo.fr \\ 2Email: mustapha.bejaoui.fsb@gmail.com \\ ${ }^{1}$ urn:lsid:zoobank.org:author:50828922-AFD9-41DF-93BC-C5F8D15264D6 \\ ${ }^{2}$ urn:1sid:zoobank.org:author:3A28BD3D-F5A6-45F5-B807-33EFC2759216 \\ ${ }^{3}$ urn:1sid:zoobank.org:author:52A050CB-4AE4-4EEF-8D5E-B508A0EC5AB2
}

\begin{abstract}
The aquatic biodiversity of springs and groundwater systems of North Africa remains largely unexplored. In an earlier field survey of Tunisian springs, a new gastropod genus, Bullaregia, was discovered as a phylogenetically independent lineage of uncertain position within the family Hydrobiidae. Here, we provide taxonomic and phylogenetic assignments for three newly collected populations of hydrobiids from springs in northern Tunisia based on morphological, anatomical and genetic (mtCOI and 18S) data. Among these and specimens of Bullaregia, major differences were observed in male and female genitalia as well as in mtCOI sequences (divergence 8.0-9.1\%). Based on these findings, we describe two new genera and three new species: Belgrandiellopsis chorfensis gen. et sp. nov., Belgrandiellopsis secunda gen. et sp. nov. and Biserta putealis gen. et sp. nov. In all our phylogenetic analyses, these three new species were well resolved as a monophyletic group together with Bullaregia tunisiensis. Unexpectedly, this clade emerged as sister to the European valvatiform genera Corbellaria and Kerkia and not to the recently discovered clade of groundwater, conchologically similar, species living in Bulgaria (Balkan Peninsula). These Tunisian species are each locally endemic and form part of a newly discovered clade which in future systematic studies could eventually be identified as a distinct hydrobiid subfamily.
\end{abstract}

Keywords. Taxonomy, phylogeny, springsnails, North Africa, Bullaregia.

Khalloufi N., Béjaoui M. \& Delicado D. 2020. Two new genera and three new subterranean species of Hydrobiidae (Caenogastropoda: Truncatelloidea) from Tunisia. European Journal of Taxonomy 648: 1-27.

https://doi.org(10.5852/ejt.2020.648

\section{Introduction}

Vast expanses of carbonate rocks in several countries of North Africa (Morocco, Algeria and Tunisia) give rise to aquifer systems under conditions of an arid or semi-arid climate (Woodward 2009). Despite 
these favourable conditions, northern African ground waters are currently under considerable stress due to climate change and/or anthropogenic factors such as land use or water pollution (Ouedraogo et al. 2016). In Tunisia, human populations have long used groundwater resurgences (springs and wells) as drinking and irrigation water. As a result, wells and captured springs can be found across the country, and these are also home to highly adapted, subterranean organisms. While more than 100 stygobiont (i.e., obligate subterranean) species have been discovered in North Africa (Gibert \& Culver 2009), information on the subterranean biodiversity of Tunisia, including those species living in springs of underground waters, is still very limited (García et al. 2010). The restricted geographic distribution of these species, often of difficult access, coupled to their small body size and convergent structures (Culver 2012) make biodiversity inventories in these ecosystems a real challenge.

Molluscs are an important component of spring and groundwater systems of North Africa (García et al. 2010). For more than a decade now, surveys of continental underground ecosystems (e.g., streams, springs and wells) in Tunisia have revealed that the most abundant and frequent mollusc species belong to the gastropod family Hydrobiidae Stimpson, 1865 (Khalloufi \& Boumaiza 2007; Khalloufi et al. 2017). Even when applying the sensu stricto definition based on molecular evidence (Wilke et al. 2001, 2013), hydrobiids are generally considered one of the most species-rich families of freshwater molluscs, and have been described as a hotspot of diversity in Northern Africa (Strong et al. 2008; Miller et al. 2018). However, the status of most Tunisian hydrobiid species is unknown, and the species richness picture of this family in Tunisia is still incomplete. Minute-sized snails from this region were previously discovered mainly from surface fresh- and brackish-waters. Based on shell morphology, most species were tentatively assigned to the non-hydrobiid genera Amnicola Gould \& Haldeman, 1840, Bythinella Moquin-Tandon, 1856 and Paludestrina D'Orbigny, 1840 (Bourguignat 1860, 1864; Debeaux 1863; Issel 1880; Letourneux \& Bourguignat 1887; Pallary 1921, 1923; Seurat 1921, 1934). Later on, according to new anatomical evidence, Boeters (1976) and Glöer et al. (2010) transferred some of these taxa to the hydrobiid genera Pseudamnicola Paulucci, 1878, Mercuria Boeters, 1971 and Hydrobia Hartmann, 1821. In the latter study (Glöer et al. 2010), new species of Pseudamnicola and Mercuria from Tunisia were also identified based on samples from museum collections. Recent molecular phylogenies (Delicado et al. 2015, 2018) have also unveiled unknown lineages within Pseudamnicola which may represent new species to science.

Sampling efforts in northern Africa, especially Tunisia, have focused little on underground water ecosystems. In Algeria and northern Tunisia, a few subterranean species with very small, conic shells (e.g., Bythinella (?) limnopsis Letourneux \& Bourguignat, 1887, B. (?) mauritanica Bourguignat, 1887, B. (?) microcochlia Letourneux \& Bourguignat, 1887, B. (?) punica Letourneux \& Bourguignat, 1887 and Hydrobia (?) nana (Terver, 1839)) were collected and described by early authors (e.g., Letourneux \& Bourguignat 1887; Pallary 1921; Terver 1839). The latter species was also reported from Zaghouan and Bulla Régia (in Tunisia). Pallary (1923) retained only this species, whose genus remains unspecified between Hydrobia and Bythinella. However, studies on these taxa have not advanced until recently. Khalloufi et al. (2017) erected a new genus, Bullaregia Khalloufi, Béjaoui \& Delicado, 2017, for the snail population living in Bulla Régia and two additional springs. Specimens of this genus resemble Bythinella and the hydrobiid genus Belgrandiella Wagner, 1928 in shell shape but bear other very distinctive anatomical features. Besides this, the sister taxa and phylogenetic position of this new genus within Hydrobiidae remain unclear (Khalloufi et al. 2017). Additional surveys are therefore necessary to discover their current diversity and evolutionary history, which are crucial to protect the snails' fragile spring habitats (Hershler et al. 2014).

The first two authors of the present paper recently collected hydrobiid snails from three subterranean water springs in northern Tunisia. Here, we provide morphological and anatomical descriptions of these three populations along with taxonomic assignments. To assess their taxonomic status, phylogenetic 
relationships and position within Hydrobiidae, we also add genetic (mitochondrial and nuclear) data for these and other recently discovered subterranean hydrobiid species from Europe (Rysiewska et al. 2016; Osikowski et al. 2017) to the previous molecular phylogeny (Khalloufi et al. 2017). This work forms part of a series of upcoming papers that will describe hydrobiid diversity in neglected and highly stressed aquatic ecosystems in Tunisia.

\section{Material and methods}

Live specimens were collected from three localities in northern Tunisia: Chorfa Spring, Province Béja, $36.865918^{\circ} \mathrm{N}, 9.363313^{\circ} \mathrm{E}, 430 \mathrm{~m}$ a.s.l.; Ettoute Spring, Province Bizerte, $36.883898^{\circ} \mathrm{N}, 9.505894^{\circ} \mathrm{E}$, 415 m a.s.l.; and Soudene Well (Menzel Bourguiba city), Province Bizerte, $37.09046^{\circ}$ N, $9.777997^{\circ}$ E, $115 \mathrm{~m}$ a.s.l. (Fig. 1). This was done within the substrate with a $300 \mu \mathrm{m}$ mesh cloth. Samples were preserved in $70 \%$ ethanol in the field and sorted in the laboratory under a MOTIC ST-39 series stereo microscope. Anatomical structures were studied under an Olympus SZX12 stereo microscope and photographed using a Keyence VHX 2000 3D Digital Microscope in combination with the program VHX-2000 Communication software ver. 2.3.5.0 (Keyence Corporation 2009-2012). The radula was
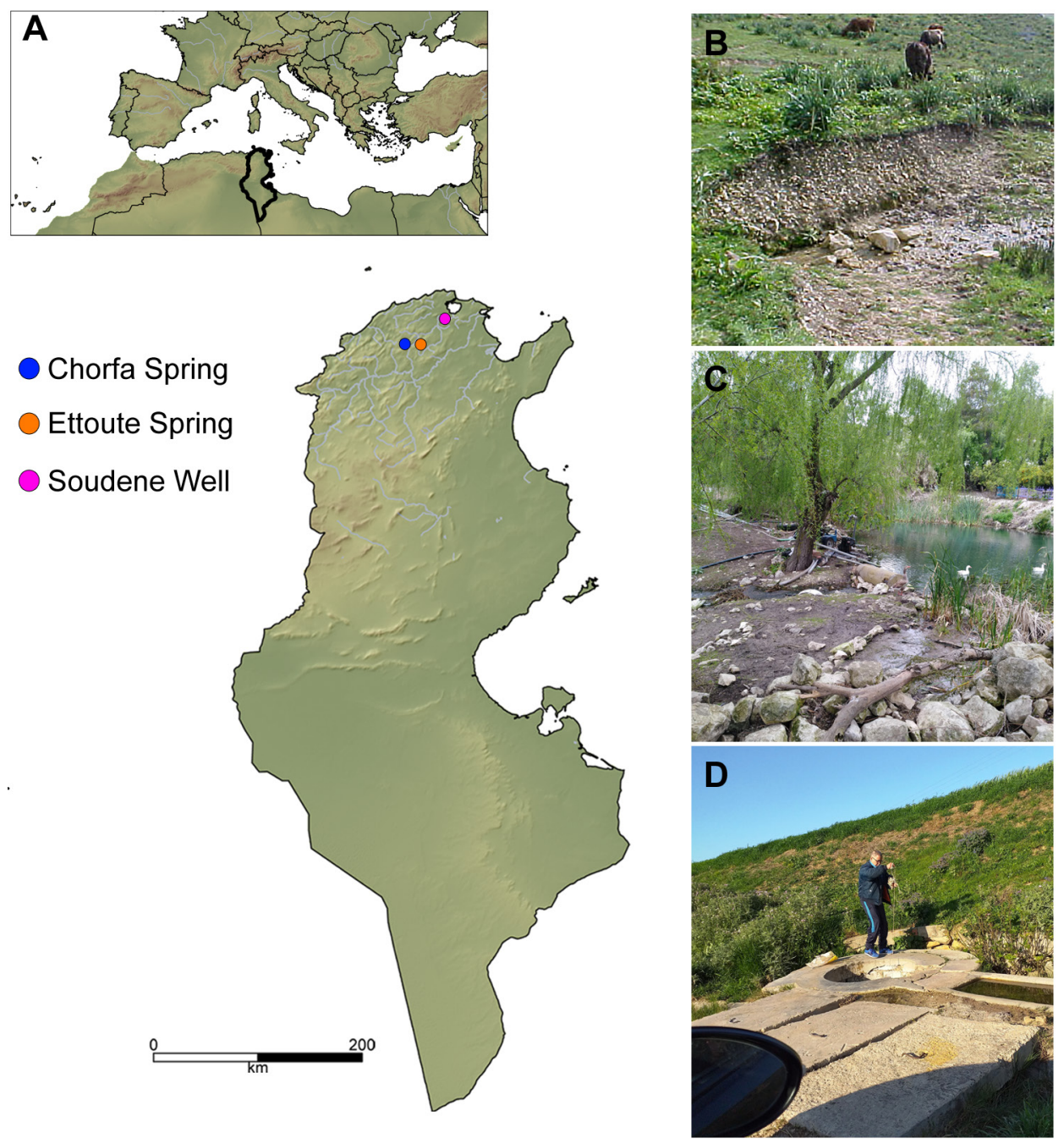

Fig. 1. Map and photographs of the collecting localities. A. Map showing the collecting localities in Tunisia. B. Chorfa Spring, Province Béja. C. Ettoute Spring, Province Bizerte. D. Soudene Well (Menzel Bourguiba city), Province Bizerte. 
extracted from the buccal mass according to the first step of a CTAB protocol for DNA isolation (Wilke et al. 2006). Then, after drying, radulae were mounted on metallic stubs and sputter-coated with gold (Balter Sputter Coater SCD004) for 50 s so that photographs could be captured with a field emission scanning electron microscope (FESEM) DSM982 Gemini (Carl Zeiss GmbH, Germany). Anatomical characteristics (genitalia, stomach, radula and gill) were described, following the standard terminology of Hershler \& Ponder (1998). Shell measurements were made with an eye-piece micrometer under the stereo microscope and consisted of length and width of the entire shell, body whorl and aperture, aperture high and width of antepenultimate and penultimate whorls (see abbreviations below). The number of specimens subjected to morphometry is indicated in the corresponding sections of the text. The 11 variables for shell dimensions and number of body whorls followed a normal distribution according to Kolmogorov-Smirnov tests and have equal variances among groups as indicated by Levene's test of homogeneity of variance. Consequently, an ANOVA could be used to test for statistical differences among these variables in the three species. These statistical calculations have been performed with the R 3.6.1 statistical environment (R Development Core Team 2019) and the R package car (Fox \& Weisberg 2019). The sex of the holotype was not examined to keep the shell intact. The holotype and some of the paratypes were deposited in the Collection of Molluscs of the Museo Nacional de Ciencias Naturales de Madrid (MNCN), Spain; the other paratypes and DNA samples were deposited in the collection of the University of Giessen Systematics and Biodiversity (UGSB) (Diehl et al. 2018) in Germany and the collection of N. Khalloufi at the Faculty of Sciences of Bizerte, University of Carthage, in Tunisia.

We genetically analysed one to two specimens from each sample. GenBank sequences from another 32 hydrobiid species (Table 1) resembling the newly discovered Tunisian populations in shell shape and/or with evidence of their close relationship to them (Khalloufi et al. 2017) were added to the genetic data matrices to assess the phylogenetic status and position of these snails within the Hydrobiidae. Genomic DNA was extracted from whole specimens using the CTAB protocol of Wilke et al. (2006). Regions of the mitochondrial cytochrome $c$ oxidase subunit I (COI) and nuclear ribosomal 18S rRNA (18S) were amplified and sequenced using the primer pairs LCO1490 (Folmer et al. 1994) and COR722b (Davis et al. 1998) for COI, and the universal metazoan $18 \mathrm{~S}$ primers (Holland et al. 1991) for 18S. Amplification conditions for COI and 18S gene fragments were those of Delicado et al. (2012) and Schreiber et al. (2012), respectively. The amplified PCR product was sequenced in an ABI 3730 XL sequencer (Life Technologies, Carlsbad, CA, USA) using a Big Dye Terminator kit ver. 3.1 (Life Technologies). New sequences were deposited in GenBank (Table 1).

Forward and reverse sequences were assembled and edited in Sequencher 4.6 (Gene Codes, Ann Arbor, MI). Sequences obtained de novo were compiled in two datasets (one per gene partition), together with sequences of other selected hydrobiid species (Table 1). Protein-coding COI sequences were unambiguously aligned in MEGA 7.0.14 (Kumar et al. 2016). The 18S fragments were aligned using MAFFT 7.402 (Katoh et al. 2002) with default settings for gap penalties [gap opening penalty (GOP) = 1.53]. Sequence divergences (uncorrected p-distances) were calculated in MEGA. Wilke et al. (2013) found little saturation within the Hydrobiidae for the COI and 18S regions and we did not, therefore, perform saturation tests. The substitution models TIM2 (Kimura 1981) $+\mathrm{I}$ (invariable sites) $+\mathrm{G}$ (rate variation among sites) and TrNef (Tamura-Nei model with equal base frequencies; Tamura \& Nei 1993) $+\mathrm{I}+\mathrm{G}$ were selected as best fitting the COI and $18 \mathrm{~S}$ datasets, respectively, in jModelTest 2.1.7 (Darriba et al. 2012) with the corrected Akaike's information criterion (AICc; Akaike 1974; Sugiura 1978; Hurvich \& Tsai 1989).

Phylogenetic relationships of the hydrobiid species examined were estimated under maximum likelihood (ML) and Bayesian inference (BI) approaches. Maximum likelihood analysis was performed using RAxML-HPC2 8 (Stamatakis 2014) on the computer cluster of the Cyber Infrastructure for the Phylogenetic Research project (CIPRES; www.phylo.org) using the GTR (Tavaré 1986) +I +G model for 
KHALLOUFI N. et al., New hydrobiid genera and species from Tunisia

Table 1 (continues on the next two pages). Taxa included in the molecular analysis with their corresponding locality names and geographical coordinates in decimal degrees, GenBank accession numbers and original references. N.D. = No data.

\begin{tabular}{|c|c|c|c|}
\hline Taxon & Locality & $\begin{array}{l}\text { GenBank \# } \\
\text { COI/18S }\end{array}$ & Original reference \\
\hline $\begin{array}{l}\text { Outgroup } \\
\text { Mercuria emiliana }\end{array}$ & $\begin{array}{l}\text { Spain, Mallorca, La Puebla } \\
(39.79111,3.10472)\end{array}$ & $\begin{array}{l}\text { AF213346 } \\
\text { AF212913 }\end{array}$ & Wilke et al. (2000) \\
\hline $\begin{array}{l}\text { Ingroup } \\
\text { Agrafia wiktori }\end{array}$ & $\begin{array}{l}\text { Greece, Evrytania, Agrafa } \\
\text { mountains, well at Sikiá } \\
(39.36861,21.63139)\end{array}$ & $\begin{array}{l}\text { JF906762 } \\
\text { JF906758 }\end{array}$ & $\begin{array}{l}\text { Szarowska \& Falniowski } \\
\text { (2011) }\end{array}$ \\
\hline Alzoniella finalina & $\begin{array}{l}\text { Italy, Liguria, Savona, Molino, } \\
\text { spring at the Porra River } \\
(44.219,8.255)\end{array}$ & $\begin{array}{l}\text { AF367650 } \\
\text { AF367686 }\end{array}$ & Wilke et al. (2001) \\
\hline Avenionia brevis & $\begin{array}{l}\text { France, Gard, spring of the } \\
\text { fountain of St.Victor-La Coste } \\
(44.057,4.636)\end{array}$ & $\begin{array}{l}\text { AF367638 } \\
\text { AF367670 }\end{array}$ & Wilke et al. (2001) \\
\hline Balkanica yankovi & $\begin{array}{l}\text { Bulgaria, Sulari and Yantra } \\
\text { villages, Izvora Cave } \\
(42.95638,25.31444)\end{array}$ & $\begin{array}{l}\text { MF179879 } \\
\text { MF179924 }\end{array}$ & Osikowski et al. (2017) \\
\hline Belgrandia thermalis & $\begin{array}{l}\text { Italy, Tuscany, Pisa, } \\
\text { S. Giuliano Terme, thermal } \\
\text { channel }(43.751,10.440)\end{array}$ & $\begin{array}{l}\text { AF367648 } \\
\text { AF367684 }\end{array}$ & Wilke et al. (2001) \\
\hline Belgrandiella kusceri & $\begin{array}{l}\text { Slovenia, spring of Rakek } \\
\text { (N.D.) }\end{array}$ & $\begin{array}{l}\text { JX970610 } \\
\text { JX970574 }\end{array}$ & Wilke et al. (2013) \\
\hline $\begin{array}{l}\text { Belgrandiellopsis chorfensis } \\
\text { gen. et sp. nov. }\end{array}$ & $\begin{array}{l}\text { Tunisia, Béja Province, Chorfa } \\
\text { Spring }(36.86592,9.36331)\end{array}$ & $\begin{array}{l}\text { MN580416 } \\
\text { MN575710 }\end{array}$ & Present study \\
\hline $\begin{array}{l}\text { Belgrandiellopsis chorfensis } \\
\text { gen. et sp. nov. }\end{array}$ & $\begin{array}{l}\text { Tunisia, Béja Province, Chorfa } \\
\text { Spring }(36.86592,9.36331)\end{array}$ & $\begin{array}{c}\text { MN580417 } \\
-\end{array}$ & Present study \\
\hline $\begin{array}{l}\text { Belgrandiellopsis secunda } \\
\text { gen. et sp. nov. }\end{array}$ & $\begin{array}{l}\text { Tunisia, Bizerte Province, } \\
\text { Ettoute Spring } \\
(36.883900,9.50589)\end{array}$ & $\begin{array}{l}\text { MN580418 } \\
\text { MN575711 }\end{array}$ & Present study \\
\hline $\begin{array}{l}\text { Belgrandiellopsis secunda } \\
\text { gen. et sp. nov. }\end{array}$ & $\begin{array}{l}\text { Tunisia, Bizerte Province, } \\
\text { Ettoute Spring } \\
(36.883900,9.50589)\end{array}$ & $\begin{array}{l}\text { MN580419 } \\
\text { MN575712 }\end{array}$ & Present study \\
\hline $\begin{array}{l}\text { Biserta putealis } \\
\text { gen. et sp. nov. }\end{array}$ & $\begin{array}{l}\text { Tunisia, Bizerte Province, } \\
\text { Soudene Well } \\
(37.09046,9.7780)\end{array}$ & $\begin{array}{l}\text { MN580420 } \\
\text { MN575713 }\end{array}$ & Present study \\
\hline Boetersiella sturmi & $\begin{array}{l}\text { Spain, Jaén, Mata Bejid, } \\
\text { La Mata Spring } \\
(37.69503,-2.49297)\end{array}$ & $\begin{array}{l}\text { MH350199 } \\
\text { MH348097 }\end{array}$ & Delicado et al. (2019) \\
\hline Boleana umbilicata & $\begin{array}{l}\text { Slovenia, Močilnik, the spring } \\
\text { of the Ljubljanica River } \\
(45.95416,14.2925)\end{array}$ & $\begin{array}{l}\text { JX982795 } \\
\text { JX982797 }\end{array}$ & $\begin{array}{l}\text { Falniowski \& Szarowska } \\
\text { (2012) }\end{array}$ \\
\hline Bullaregia tunisiensis & $\begin{array}{l}\text { Tunisia, Province Béja, a } \\
\text { spring in Djebba } \\
(36.47125,9.09972)\end{array}$ & $\begin{array}{l}\text { KX821683 } \\
\text { MN575709 }\end{array}$ & $\begin{array}{l}\text { Khalloufi et al. (2017) } \\
\text { Present study }\end{array}$ \\
\hline
\end{tabular}


Table 1 (continued).

\begin{tabular}{|c|c|c|c|}
\hline Taxon & Locality & $\begin{array}{l}\text { GenBank \# } \\
\text { COI/18S }\end{array}$ & Original reference \\
\hline Bullaregia tunisiensis & $\begin{array}{l}\text { Tunisia, Province Béja, a } \\
\text { spring in Djebba } \\
(36.47125,9.09972)\end{array}$ & $\begin{array}{c}\text { MN580415 } \\
-\end{array}$ & Present study \\
\hline Cavernisa zaschevi & $\begin{array}{l}\text { Bulgaria, Tserovo, Vodnata } \\
\text { Cave }(45.95417,14.2925)\end{array}$ & $\begin{array}{l}\text { MF179895 } \\
\text { MF179931 }\end{array}$ & Osikowski et al. (2017) \\
\hline Corbellaria celtiberica & $\begin{array}{l}\text { Spain, Soria, Manubles River } \\
(41.60990,-1.95580)\end{array}$ & $\begin{array}{l}\text { MH350207 } \\
\text { MH348099 }\end{array}$ & Delicado et al. (2019) \\
\hline Dalmatinella fluviatilis & $\begin{array}{l}\text { Croatia, Jankovica Buk } \\
\text { waterfalls, Zrmanja River } \\
(44.20272,15.72136)\end{array}$ & $\begin{array}{l}\text { KC344541 } \\
\text { KC344539 }\end{array}$ & $\begin{array}{l}\text { Falniowski \& Szarowska } \\
\text { (2013) }\end{array}$ \\
\hline Daphniola graeca & $\begin{array}{l}\text { Greece, Dafne Spring } \\
(39.89108,22.60722)\end{array}$ & $\begin{array}{l}\text { EF070618 } \\
\text { EF070624 }\end{array}$ & Szarowska (2006) \\
\hline Deganta azarum & $\begin{array}{l}\text { Spain, Asturias, Borondes, } \\
\text { La Fontona Spring } \\
(43.33189,-5.98506)\end{array}$ & $\begin{array}{l}\text { MH350208 } \\
\text { MH348100 }\end{array}$ & Delicado et al. (2019) \\
\hline Devetakia mandrica & $\begin{array}{l}\text { Bulgaria, Chavdartsi, Mandrata } \\
\text { Cave }(43.24222,24.96917)\end{array}$ & $\begin{array}{l}\text { MF179891 } \\
\text { MF179927 }\end{array}$ & Osikowski et al. (2017) \\
\hline Devetakiola devetakium & $\begin{array}{l}\text { Bulgaria, Prevala, spring near } \\
\text { Vreloto Cave } \\
(43.46944,22.86111)\end{array}$ & $\begin{array}{l}\text { MF179901 } \\
\text { MF179935 }\end{array}$ & Osikowski et al. (2017) \\
\hline Fissuria boui & $\begin{array}{l}\text { France, Alpes Maritimes, } \\
\text { Peymeinade, spring near La } \\
\text { Prouveresse } \\
(43.64279,6.88735)\end{array}$ & $\begin{array}{l}\text { AF367654 } \\
\text { AF367690 }\end{array}$ & Wilke et al. (2001) \\
\hline $\begin{array}{l}\text { Graecoarganiella } \\
\text { parnassiana }\end{array}$ & $\begin{array}{l}\text { Greece, Parnassus mountains, } \\
\text { south of Eptalofos, small } \\
\text { spring }(38.59278,22.5039)\end{array}$ & $\begin{array}{l}\text { JN202348 } \\
\text { JN202341 }\end{array}$ & $\begin{array}{l}\text { Falniowski \& Szarowska } \\
\text { (2011) }\end{array}$ \\
\hline Graziana alpestris & $\begin{array}{l}\text { Italy, Liguria, Savona, Molino, } \\
\text { spring at the Porra River } \\
(44.219,8.255)\end{array}$ & $\begin{array}{l}\text { AF367641 } \\
\text { AF367673 }\end{array}$ & Wilke et al. (2001) \\
\hline Grossuana codreanui & $\begin{array}{l}\text { Romania, a spring close to the } \\
\text { lake Techirghiol } \\
(43.99361,28.54611)\end{array}$ & $\begin{array}{l}\text { EF061919 } \\
\text { EF061916 }\end{array}$ & Szarowska et al. (2007) \\
\hline Hauffenia tellinii & $\begin{array}{l}\text { Italy, Friuli-Venetia Julia, } \\
\text { Gorizia, spring of the Isonzo } \\
\text { River near Sagrado } \\
(45.8743,13.4856)\end{array}$ & $\begin{array}{l}\text { AF367640 } \\
\text { AF367672 }\end{array}$ & Wilke et al. (2001) \\
\hline Horatia klecakiana & $\begin{array}{l}\text { Croatia, N of Kučice, Studenci } \\
\text { spring, in the valley of the } \\
\text { Cetina River } \\
(43.44481,16.80708)\end{array}$ & $\begin{array}{l}\text { KJ159128 } \\
\text { KJ159127 }\end{array}$ & $\begin{array}{l}\text { Szarowska \& Falniowski } \\
\text { (2014) }\end{array}$ \\
\hline Islamia globulus & $\begin{array}{l}\text { Spain, Huesca, Gistain, Grima } \\
\text { Spring }(42.58660,-0.31766)\end{array}$ & $\begin{array}{l}\text { MH350222 } \\
\text { MH348103 }\end{array}$ & Delicado et al. (2019) \\
\hline
\end{tabular}


KHALLOUFI N. et al., New hydrobiid genera and species from Tunisia

Table 1 (continued).

\begin{tabular}{|c|c|c|c|}
\hline Taxon & Locality & $\begin{array}{c}\text { GenBank \# } \\
\text { COI } / 18 S\end{array}$ & Original reference \\
\hline Kerkia kusceri & $\begin{array}{l}\text { Slovenia, Ivancna Gorica, Cave } \\
\text { Krška jama }(45.89,14.7711)\end{array}$ & $\begin{array}{l}\text { KY087867 } \\
\text { KY087833 }\end{array}$ & Rysiewska et al. (2017) \\
\hline Pauluccinella minima & $\begin{array}{l}\text { Italy, S. Egidio, Lago di } \\
\text { Piediluco }(42.52925,12.7474)\end{array}$ & $\begin{array}{l}\text { JX970612 } \\
\text { JX970578 }\end{array}$ & Wilke et al. (2013) \\
\hline Pontobelgrandiella angelovi & $\begin{array}{l}\text { Bulgaria, a spring in Tvarditsa } \\
(42.70556,25.89778)\end{array}$ & $\begin{array}{l}\text { KU496965 } \\
\text { MF179938 }\end{array}$ & $\begin{array}{l}\text { Rysiewska et al. (2016) } \\
\text { Osikowski et al. (2017) }\end{array}$ \\
\hline Pseudamnicola lucensis & $\begin{array}{l}\text { Italy, Tuscany, Bagni di Lucca, } \\
\text { Bagni Caldi, thermal spring } \\
(44.007,10.585)\end{array}$ & $\begin{array}{l}\text { AF367651 } \\
\text { AF367687 }\end{array}$ & Wilke et al. (2001) \\
\hline Radomaniola callosa & $\begin{array}{l}\text { Italy, Abruzzo, Caramanico } \\
\text { Terme, stream on the left bank } \\
\text { of Orfento River } \\
(42.1571,14.0167)\end{array}$ & $\begin{array}{l}\text { AF367649 } \\
\text { AF367685 }\end{array}$ & Wilke et al. (2001) \\
\hline Sadleriana fluminensis & $\begin{array}{l}\text { Croatia, Jadro River at Solin } \\
\text { near Split }(43.5453,16.48780)\end{array}$ & $\begin{array}{l}\text { AF367647 } \\
\text { AF367683 }\end{array}$ & Wilke et al. (2001) \\
\hline Stoyanovia stoyanovi & $\begin{array}{l}\text { Bulgaria, Bezhanovo village, } \\
\text { Parnitsite Cave } \\
(43.22889,24.38861)\end{array}$ & $\begin{array}{l}\text { MF179902 } \\
\text { MF179936 }\end{array}$ & Osikowski et al. (2017) \\
\hline Trichonia kephalovrissonia & $\begin{array}{l}\text { Greece, spring in Thérmon } \\
(38.57294,21.66622)\end{array}$ & $\begin{array}{l}\text { EF070619 } \\
\text { EF070630 }\end{array}$ & Szarowska (2006) \\
\hline
\end{tabular}

each partition and a rapid bootstrapping algorithm. Node support was assessed through 1000 bootstrap (BS) pseudoreplicates. Bayesian inference analysis was conducted in MrBayes 3.2.6 (Ronquist et al. 2012) using the substitution models selected through jModelTest. Markov chain Monte Carlo (MCMC) methods were run with random starting trees and 4 chains for 5000000 generations, sampling one generation every 1000 sampling points. After verifying chain convergence (i.e., average standard deviations of split frequencies $<0.01$ ), the first $10 \%$ of the samples was discarded as burn-in. The robustness of the inferred trees was evaluated by Bayesian posterior probabilities (BPPs). Tree topologies and branch supports were visualized in FigTree 1.4.3 (Rambaut 2010).

\section{Abbreviations}

Shell measurements

$\mathrm{AH}=$ aperture height

$\mathrm{AL}=$ aperture length

$\mathrm{AW}=$ aperture width

LBW $=$ length of the body whorl

NSW $=$ number of spire whorls

$\mathrm{SL}=$ shell length

SW $=$ shell width

WAW $=$ width of antepenultimate whorl

WBW $=$ width of body whorl

WPW $=$ width of penultimate whorl 
Anatomy

$\mathrm{Ag}=$ albumen gland

$\mathrm{Bc}=$ bursa copulatrix

$\mathrm{Bd}=$ bursal duct

$\mathrm{Bl}=$ basal lobe

$\mathrm{Bp}=$ basal portion of penis

$\mathrm{Cg}=$ capsule gland

$\mathrm{Ct}=$ ctenidium

$\mathrm{Dl}=$ distal lobe

$\mathrm{Dp}=$ distal portion of penis

$\mathrm{Op}=$ oviduct pouch

Os $=$ osphradium

$\mathrm{Ov}=$ renal oviduct

Pf $=$ penial filament (i.e., structure apart from lobes)

$\mathrm{SR} 1=$ distal seminal receptacle

$\mathrm{SR} 2=$ proximal seminal receptacle

$\mathrm{Tp}=$ terminal papilla

\title{
Results
}

The combined alignment of the COI ( $648 \mathrm{bp}$ ) and $18 \mathrm{~S}$ (468 bp) sequences yielded $1116 \mathrm{bp}$. Average base frequencies for $\mathrm{COI}$ were $30.8 \% \mathrm{~A}, 13.0 \% \mathrm{C}, 11.2 \% \mathrm{G}$ and $45 \% \mathrm{~T}$. Based on the combined dataset, $\mathrm{ML}$ and $\mathrm{BI}$ trees agreed in depicting a well-supported $(\mathrm{BS}=100 \%, \mathrm{BPP}=1)$ monophyletic clade, informally referred to herein as the 'Tunisian clade', comprising the Tunisian populations examined here and Bullaregia tunisiensis. Each of the Tunisian populations formed a well-resolved subclade within this clade (Fig. 2). Uncorrected pairwise sequence divergences among representatives of this clade ranged from $7.9-9.3 \%$ for COI and were $0 \%$ for $18 \mathrm{~S}$. These results are similar to those observed among other genera of our phylogenetic study (e.g., 6.2-10\% COI and $0 \% 18 \mathrm{~S}$ among the subterranean genera from Bulgaria). Phylogenetic relationships within the Tunisian clade were well supported between populations from Chorfa and Ettoute Springs $(\mathrm{BS}=86 \%, \mathrm{BPP}=0.96)$. The remaining relationships within this clade were weakly supported. $\mathrm{BI}$ analysis resolved $(\mathrm{BPP}=1)$ the Tunisian clade as sister to the species Corbellaria celtiberica Girardi \& Boeters, 2012 from the Iberian Peninsula and Kerkia kusceri (Bole, 1961) from the Balkans. In both analyses, all these species weakly grouped with the subterranean hydrobiid genera from Bulgaria (Fig. 2).

\author{
Phylum Mollusca Cuvier, 1795 \\ Class Gastropoda Cuvier, 1795 \\ Superorder Caenogastropoda Cox, 1960 \\ Superfamily Truncatelloidea Gray, 1840 \\ Family Hydrobiidae Stimpson, 1865 \\ Genus Belgrandiellopsis gen. nov. \\ urn:lsid:zoobank.org:act:6073FD1F-91C0-4C93-B1CB-09AA54B1FABD
}

\section{Type species}

Belgrandiellopsis chorfensis sp. nov., by present designation.

\section{Diagnosis}

Shell small (maximum length slightly exceeding $2 \mathrm{~mm}$ ), ovate-conic to elongate-conic, with obtuse and planispiral protoconch; whorls slightly convex, with weak sutures. Penultimate whorl tall relative 
to remaining whorls. Aperture pyriform to ovate, complete, angled on top and often fused to the body whorl. Umbilicus closed by inner lip. Operculum corneous, whitish to yellowish, thin, pliable, elongateellipsoidal, spiral, paucispiral, with submarginal nucleus. One pair of small triangular basal cusps on radular central tooth. Ctenidium well developed. Stomach with a small gastric (posterior) caecum. Presence of bursa copulatrix; unpigmented renal oviduct with a spherical pouch at the end of the coiled section; two seminal receptacles. Penis attached to the neck behind the right eye with two glandular lobes distantly positioned, each of them bearing a terminal papilla.

\section{Etymology}

Refers to the shell similarities with the hydrobiid genus Belgrandiella; gender feminine.

\section{Remarks}

Belgrandiellopsis gen. nov. differs from the conchologically similar genus Belgrandiella in having a strap-like penis with two glandular lobes, two seminal receptacles and one pair of basal cusps on the central radular teeth (for comparison see Szarowska 2006); from the closely related genus Bullaregia according to the presence of two lobes on the inner edge of the penis, smaller gastric caecum, shorter

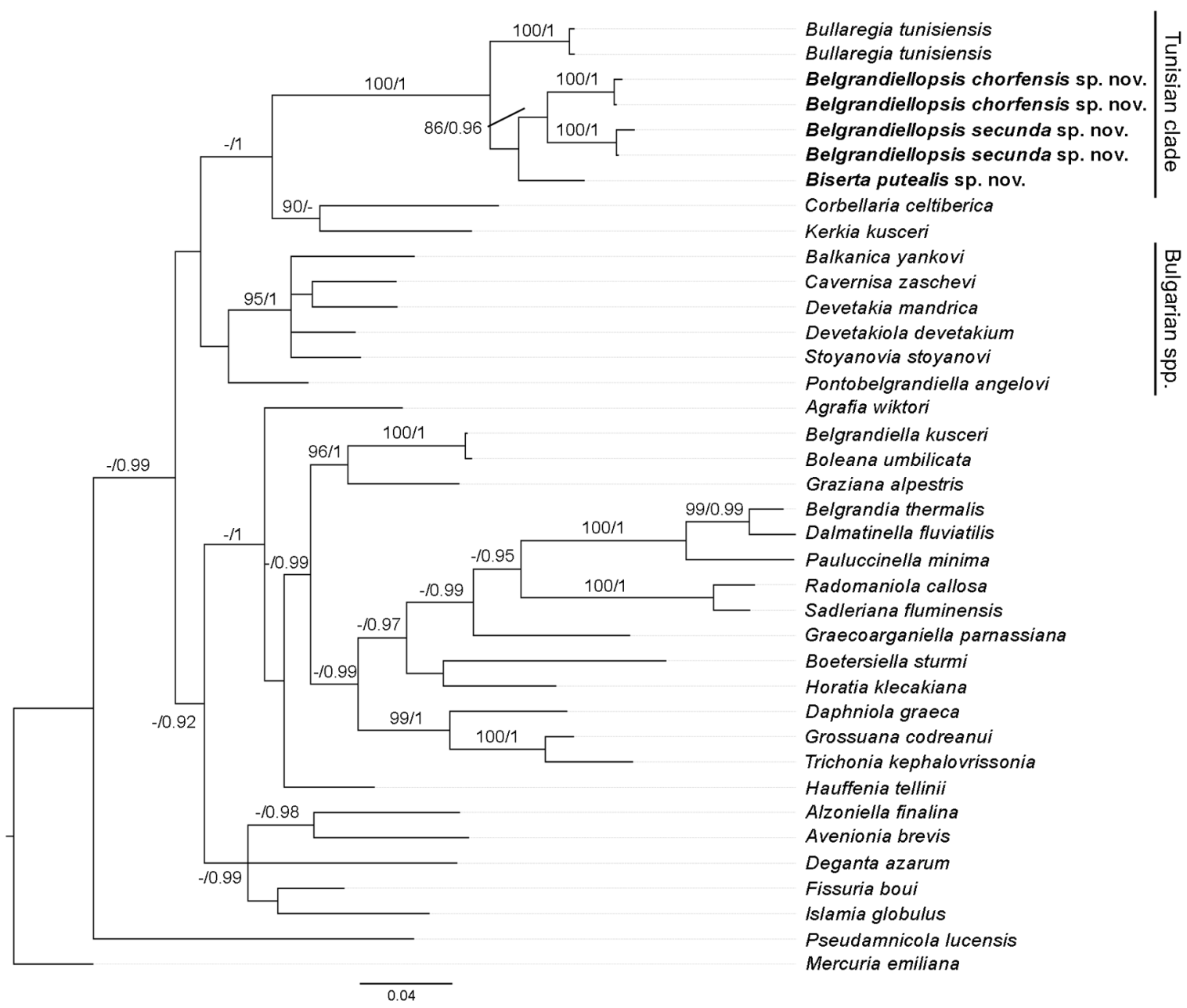

Fig. 2. Bayesian inference based on the $\mathrm{COI}$ and $18 \mathrm{~S}$ datasets. Bootstrap values and BPP's for branches are given when $\geq 70 \%$ and $\geq 0.90$, respectively. Scale $b a r=$ expected change per site. 
bursal duct, larger bursa copulatrix and in having generally a smaller number of cusps on radular teeth (see Khalloufi et al. 2017); and from Biserta gen. nov. according to its smaller shell umbilicus, longer penis with a larger distance between penial lobes, slightly smaller bursa copulatrix and longer bursal duct (Figs 4, 6, 8). Belgrandiellopsis gen. nov. differs in its mean COI divergence by $9.3 \%$ and $8.4 \%$ from the closely related Bullaregia and Biserta gen. nov., respectively, and by 17.6\% from Belgrandiella.

Belgrandiellopsis chorfensis gen. et sp. nov. urn:lsid:zoobank.org:act:2C5704A1-BDFC-41BA-86C6-CF17A999BBC8

Figs 3-4

\section{Diagnosis}

Shell 1.45 to $1.65 \mathrm{~mm}$ high, elongate-conic, brown-yellowish; central radular tooth formula (5)4-C4(5)/1-1; bursa copulatrix ovoid, with the same length as the duct; two seminal receptacles, SR1 more developed and pedunculated, SR2 small, globular, located near loop before the pouch; prostate gland bean shaped, ovate in section, folded; penis cylindrical, slender, distal end tapered, unpigmented, with two lobes distantly positioned; basal lobe more developed. Distinguished from other closely related species by $>8.0 \%$ for COI.

\section{Etymology}

A geographic epithet referring to Chorfa Spring, where the species was found.

\section{Type material}

\section{Holotype}

TUNISIA - 1 spec. (sex unknown, preserved in ethanol 80\%); Béja Province, ca $15 \mathrm{~km}$ west of

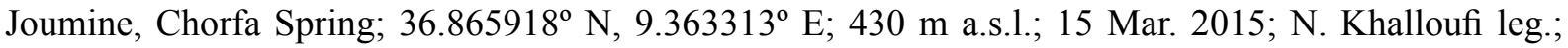
MNCN 15.05/200097H

\section{Paratypes}

TUNISIA - 5 specs (preserved in ethanol 80\%); same collection data as for holotype; MNCN 15.05/200097P • 40 specs (preserved in ethanol 80\%, 2 우, 2 ô ${ }^{\lambda}$ dissected and 2 specs processed for DNA sequencing); same collection data as for holotype; UGSB $17663 \cdot 150$ specs (preserved in ethanol 95\%, ca 70 individuals dissected); same collection data as for holotype; Khalloufi's collection.

\section{Type locality}

TUNISIA: Béja Province, ca $15 \mathrm{~km}$ west of Joumine, Chorfa Spring, $36.865918^{\circ} \mathrm{N}, 9.363313^{\circ} \mathrm{E}, 430 \mathrm{~m}$ a.s.l.

\section{Description}

MeAsurements. Holotype: $\mathrm{SL}=1.61 \mathrm{~mm}, \mathrm{SW}=0.88 \mathrm{~mm}, \mathrm{SL} / \mathrm{SW}=1.83, \mathrm{AH}=0.66 \mathrm{~mm}$, SL-LBW $=$ $0.49 \mathrm{~mm}, \mathrm{WBW}=0.84 \mathrm{~mm}, \mathrm{AL}=0.78 \mathrm{~mm}, \mathrm{AW}=0.53 \mathrm{~mm}, \mathrm{WPW}=0.64 \mathrm{~mm}, \mathrm{WAW}=0.10 \mathrm{~mm}$.

SHELl. Elongate-conic, brown to yellowish, with 4-4.5 whorls (Fig. 3A-D; Table 2). Protoconch (Fig. 3G) obtuse, near planispiral, about 1.2 whorls, diameter about $350 \mu \mathrm{m}$; protoconch microsculpture granulated. Teleoconch whorls weakly convex, separated by deep sutures; body whorl occupying $2 / 3$ of total shell length. Aperture about $40 \%$ of total shell length, pyriform, strongly angled on the top and narrowly disjunct from parietal wall of body whorl; inner lip thin but thicker than outer lip; peristome margin (Fig. 3B) straight. Umbilicus narrow and closed by inner lip. 
Operculum. As for genus, thin, flat, whitish (nuclear region slightly yellowish), with ca 2 whorls (Fig. 3E-F); attachment scar area, oval and located near nucleus.

RADULA. Length intermediate ( $20 \%$ total maximum shell length), having about 80 rows of teeth. Central tooth (Fig. 3H-I) formula (5)4-C-4(5)/1-1, cutting edge concave; basal tooth face M-shaped and relatively narrow wings; basal tongue broadly $\mathrm{V}$-shaped, about equal to lateral margins; a single small pair of basal cusps, triangular. Lateral teeth (Fig. 3J) formula (4)3-C-5; central cusp large, narrow, pointed. Inner marginal teeth (Fig. 3H) having 25-27 sharp cusps. Outer marginal teeth having 20-22 sharp cusps.

Pigmentation AND ANATOMY. Visceral coil brown-yellowish, showing by transparency the gonad; pallial coil nearly pale with traces of pigment on the roof, anteriorly to edge of mantle; foot and area between tentacles slightly pigmented (Fig. 4C). Black small eyes surrounded by brownish pigment. Snout with

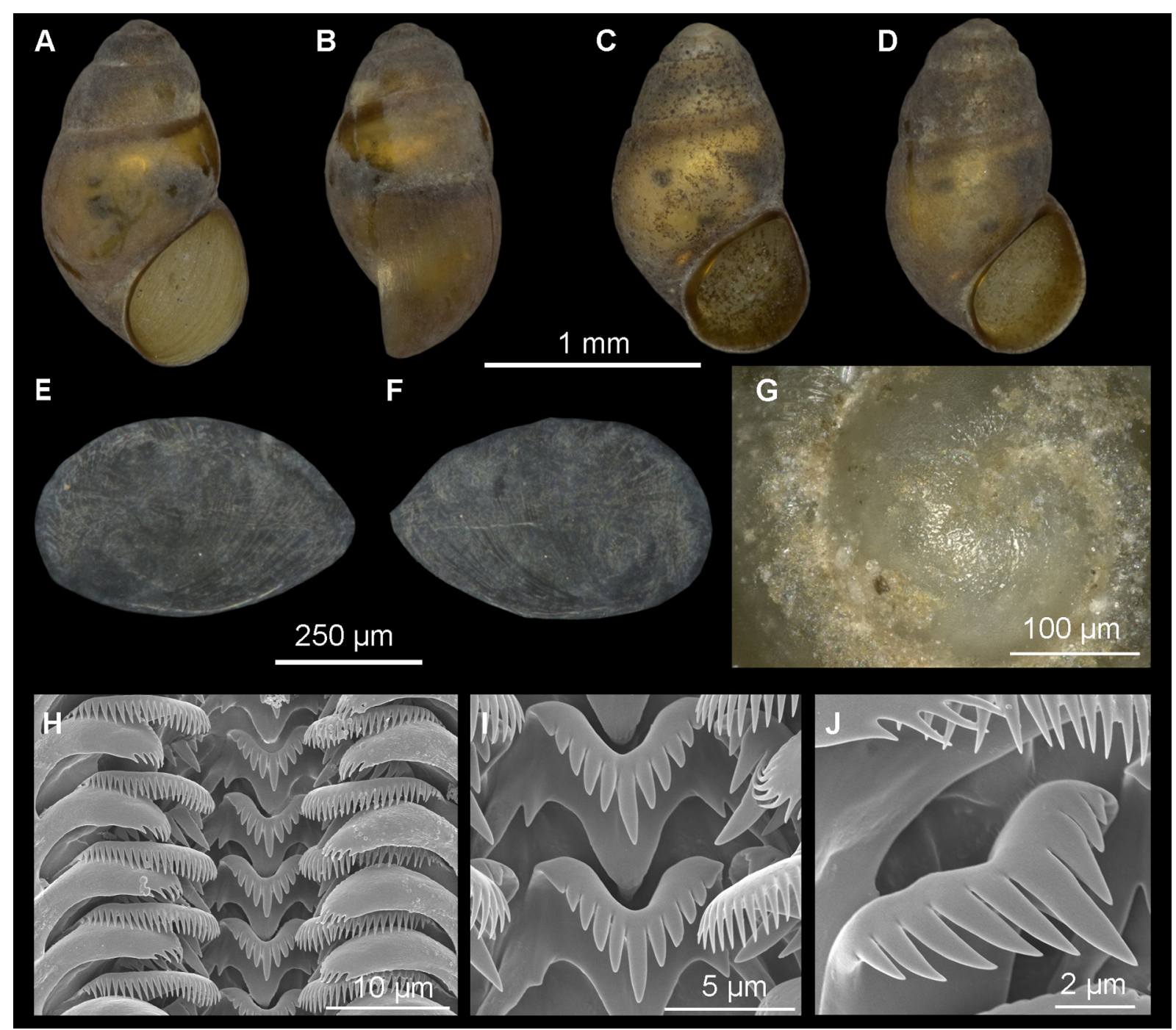

Fig. 3. Shells, opercula and radulae, Belgrandiellopsis chorfensis gen. et sp. nov. A-D. Shells. A-B. Holotype, MNCN 15.05/200097H. C-D. Paratypes, MNCN 15.05/200097P. E-F. Operculum $(\mathrm{E}=$ inner side; $\mathrm{F}=$ outer side). G. Detailed microsculpture of protoconch. $\mathbf{H}-\mathbf{J}$. Radulae. H. Portion of radula ribbon. I. Central radular teeth. J. Lateral teeth. 
medium distal lobation. Tentacles parallel-side, distal end nonexpanded. Ctenidium filaments about 12, small, taller than wide. Osphradium whitish, ellipsoidal, positioned centrally along ctenidium (Fig. 4A). Stomach slightly longer than wide with a small gastric (posterior) caecum (Fig. 4B); rectum wide, folded and S-shaped.

Male genitalia. Penis cylindrical (Fig. 4D-E), medium sized, unpigmented, slender, slightly folded; filament short, tapering; basal portion intermediate in width, shorter than distal portion, attached centrally to the head well behind the eyes; penis having two developed lobes located relatively far from one another on opposite sides of penis; both lobes with distal papilla, showing by transparency

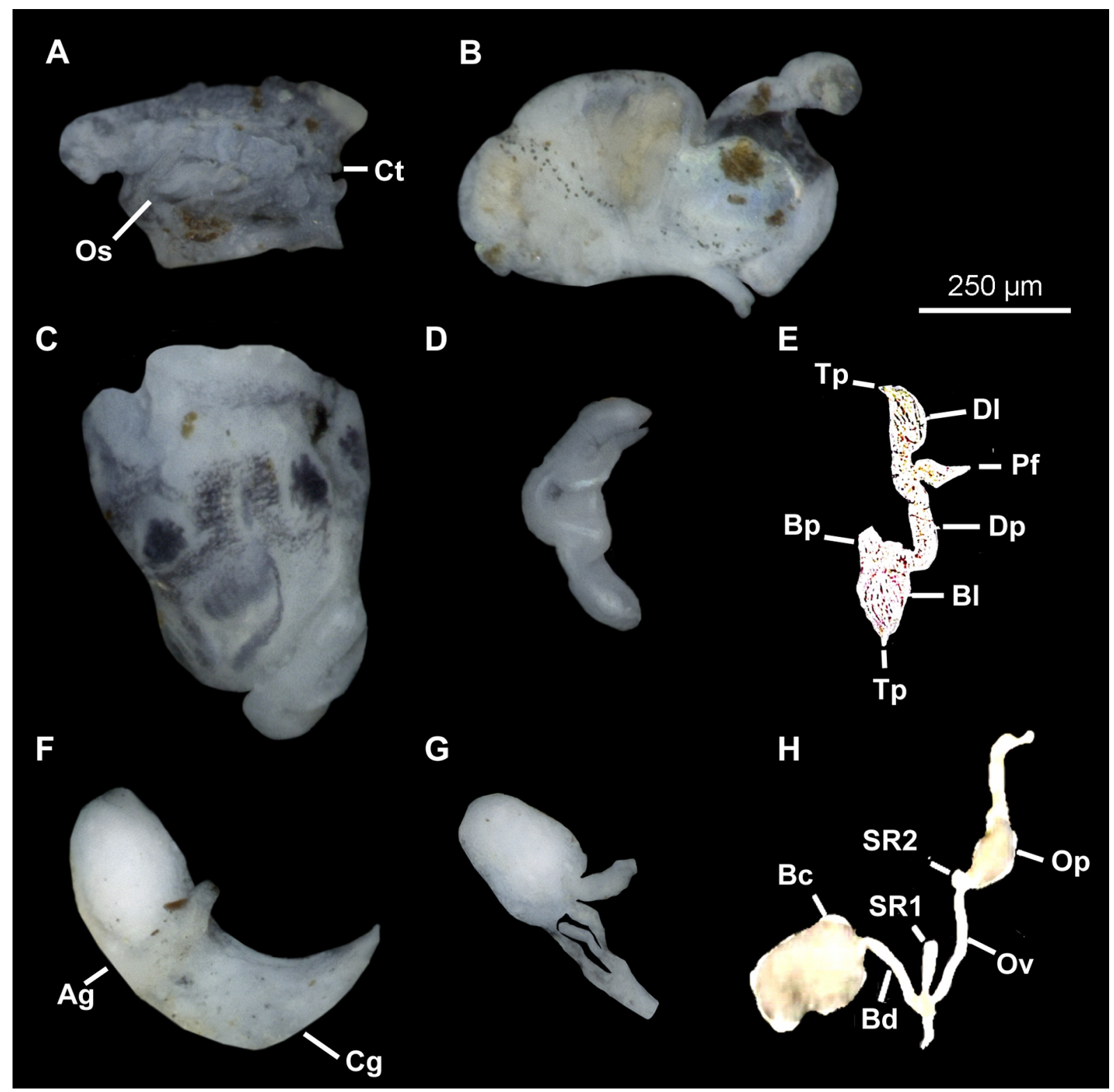

Fig. 4. Anatomy of Belgrandiellopsis chorfensis gen. et sp. nov. (paratypes, UGSB 17663). A. Ctenidium and osphradium. B. Stomach. C. Head of male and penis. D. Detail of penis and penis lobes (observed by transmitted light in E). F. Pallial oviduct. G. Bursa copulatrix and seminal receptacles. H. Ditto, observed by transmitted light. Anatomical abbreviations explained in the text. 
Table 2. Shell measurements (in $\mathrm{mm}$ ) for the species. 1. Belgrandiellopsis chorfensis gen. et sp. nov. from Chorfa Spring. 2. B. secunda gen. et sp. nov. from Ettoute Spring. 3. Biserta putealis gen. et sp. nov. from Soudene Well. *** $\mathrm{P}<0.001 ; * * \mathrm{P}<0.01 ; * \mathrm{P}<0.05$; ns, not significant.

\begin{tabular}{|c|c|c|c|c|}
\hline & $\begin{array}{c}1 \\
\text { Mean } \pm \text { SD; CV } \\
(\text { Min-Max }) \\
(\mathrm{n}=13)\end{array}$ & $\begin{array}{c}\mathbf{2} \\
\text { Mean } \pm \text { SD; CV } \\
(\text { Min-Max }) \\
(\mathrm{n}=12)\end{array}$ & $\begin{array}{c}3 \\
\text { Mean } \pm \text { SD; CV } \\
(\text { Min-Max }) \\
(\mathrm{n}=10)\end{array}$ & ANOVA \\
\hline $\mathrm{SL}$ & $\begin{array}{c}1.54 \pm 0.05 ; 0.04 \\
(1.45-1.63)\end{array}$ & $\begin{array}{c}1.92 \pm 0.12 ; 0.06 \\
(1.73-2.11)\end{array}$ & $\begin{array}{c}1.74 \pm 0.10 ; 0.06 \\
(1.60-2.00)\end{array}$ & $\mathrm{F}_{2,32}=49.55^{* * *}$ \\
\hline SW & $\begin{array}{l}0.84 \pm 0.03 ; 0.04 \\
\quad(0.79-0.89)\end{array}$ & $\begin{array}{c}1.10 \pm 0.07 ; 0.06 \\
(1.02-1.28)\end{array}$ & $\begin{array}{c}0.97 \pm 0.05 ; 0.05 \\
\quad(0.87-1.04)\end{array}$ & $\mathrm{F}_{2,32}=82.64^{* * *}$ \\
\hline SL/SW & $\begin{array}{c}1.84 \pm 0.06 ; 0.04 \\
\quad(1.75-1.95)\end{array}$ & $\begin{array}{c}1.74 \pm 0.06 ; 0.04 \\
(1.65-1.87)\end{array}$ & $\begin{array}{c}1.79 \pm 0.10 ; 0.05 \\
(1.63-1.95)\end{array}$ & $\mathrm{F}_{2,32}=4.76^{*}$ \\
\hline $\mathrm{AH}$ & $\begin{array}{l}0.66 \pm 0.04 ; 0.07 \\
\quad(0.59-0.72)\end{array}$ & $\begin{array}{c}0.87 \pm 0.06 ; 0.07 \\
\quad(0.75-0.95)\end{array}$ & $\begin{array}{l}0.80 \pm 0.09 ; 0.11 \\
\quad(0.69-0.98)\end{array}$ & $\mathrm{F}_{2,32}=34.85^{* * *}$ \\
\hline SL-LBW & $\begin{array}{c}0.46 \pm 0.04 ; 0.08 \\
\quad(0.40-0.53)\end{array}$ & $\begin{array}{c}0.53 \pm 0.06 ; 0.10 \\
\quad(0.45-0.64)\end{array}$ & $\begin{array}{c}0.53 \pm 0.08 ; 0.15 \\
\quad(0.42-0.70)\end{array}$ & $\mathrm{F}_{2,32}=5.85^{* *}$ \\
\hline WBW & $\begin{array}{l}0.80 \pm 0.03 ; 0.04 \\
\quad(0.75-0.85)\end{array}$ & $\begin{array}{c}1.03 \pm 0.05 ; 0.05 \\
(0.94-1.14)\end{array}$ & $\begin{array}{l}0.90 \pm 0.04 ; 0.05 \\
\quad(0.83-0.97)\end{array}$ & $\mathrm{F}_{2,32}=97.23^{* * *}$ \\
\hline $\mathrm{AL}$ & $\begin{array}{l}0.70 \pm 0.03 ; 0.05 \\
\quad(0.68-0.78)\end{array}$ & $\begin{array}{c}0.93 \pm 0.03 ; 0.03 \\
\quad(0.87-0.99)\end{array}$ & $\begin{array}{l}0.84 \pm 0.05 ; 0.06 \\
\quad(0.77-0.93)\end{array}$ & $\mathrm{F}_{2,32}=106.4^{* * *}$ \\
\hline AW & $\begin{array}{c}0.50 \pm 0.03 ; 0.07 \\
\quad(0.44-0.56)\end{array}$ & $\begin{array}{c}0.68 \pm 0.06 ; 0.09 \\
\quad(0.60-0.80)\end{array}$ & $\begin{array}{c}0.60 \pm 0.04 ; 0.07 \\
\quad(0.54-0.70)\end{array}$ & $\mathrm{F}_{2,32}=44.17^{* * *}$ \\
\hline WPW & $\begin{array}{c}0.60 \pm 0.03 ; 0.05 \\
\quad(0.57-0.66)\end{array}$ & $\begin{array}{c}0.72 \pm 0.04 ; 0.06 \\
\quad(0.65-0.78)\end{array}$ & $\begin{array}{l}0.67 \pm 0.06 ; 0.09 \\
\quad(0.60-0.78)\end{array}$ & $\mathrm{F}_{2,32}=19.69^{* * *}$ \\
\hline WAW & $\begin{array}{l}0.10 \pm 0.02 ; 0.19 \\
\quad(0.06-0.13)\end{array}$ & $\begin{array}{c}0.11 \pm 0.02 ; 0.20 \\
\quad(0.08-0.14)\end{array}$ & $\begin{array}{l}0.11 \pm 0.03 ; 0.24 \\
\quad(0.08-0.17)\end{array}$ & $\mathrm{F}_{2,32}=1.22^{\mathrm{ns}}$ \\
\hline NSW & $\begin{array}{c}4.13 \pm 0.17 ; 0.04 \\
\quad(4.00-4.50)\end{array}$ & $\begin{array}{c}4.29 \pm 0.23 ; 0.05 \\
\quad(4.00-4.50)\end{array}$ & $\begin{array}{c}4.18 \pm 0.17 ; 0.04 \\
\quad(4.00-4.50)\end{array}$ & $\mathrm{F}_{2,32}=2.19^{\mathrm{ns}}$ \\
\hline
\end{tabular}

tubular glands distal; basal lobe larger, thumb-like, not pedunculated; distal lobe smaller, pyriform, pedunculated. Prostate gland small, close, bean-shaped, longer than wide $(0.2 \mathrm{~mm} / 0.10 \mathrm{~mm})$. Posterior vas deferens developed in its median portion with several loops (about $4 \mathrm{~mm}$ in length and $0.01 \mathrm{~mm}$ in section); seminal vesicle yellowish. Pallial vas deferens straight, narrow, whitish, unciliated, entering the posteroventral portion of gland and exiting from its anterior end. 
Female Genitalia. Ovary simple, with stalked lobes, occupying near $40 \%$ of visceral coil. Distal female genitalia occupying near $70 \%$ of pallial roof; albumen gland approximately as long as capsule gland (Fig. 4F). Bursa copulatrix ovoid to pyriform, having a duct as long as bursal length. Renal oviduct unpigmented, coiled, having a spherical pouch at the end of the coiled section. Two seminal receptacles; SR1 medium sized, elongate, pedunculate, joining renal oviduct just above the insertion point with bursal duct; SR2 small, globular, sessile, located on renal oviduct near loop before pouch (Fig. 4G-H).

\section{Ecology and distribution}

Belgrandiellopsis chorfensis gen. et sp. nov. was found in a small spring near a rural settlement called Chorfa between the cities of Mateur and Beja, in northern Tunisia. It is known only from the type locality and some springs in this region (e.g., Ain Elgaid Spring). The specimens were found on the bottom and attached to the underside (algae-side) of gravel and stones. Density decreased drastically downstream from the water resurgence. Pseudamnicola sp. was the only co-occurring gastropod species.

\section{Remarks}

Differentiated from its congener according to its smaller shell size (Table 2), smaller number of lateral cusps on the central radular teeth, larger penis and slightly smaller bursa copulatrix. Belgrandiellopsis chorfensis gen. et sp. nov. also differs in its mean COI divergence by $8.4 \%$ from $B$. secunda gen. et sp. nov.

Belgrandiellopsis secunda gen. et sp. nov.

urn:1sid:zoobank.org:act:D3A45301-6EBE-4086-A740-FEFBE9328FB5

Figs 5-6

\section{Diagnosis}

Shell 1.7 to $2.1 \mathrm{~mm}$ high, elongate-conic, brown-yellowish; central radular tooth formula (6)5-C5(6)/1-1; bursa copulatrix ovoid with short duct; two seminal receptacles, SR1 more developed and pedunculated, SR2 very small, globular, located near loop before the pouch; prostate gland bean shaped, ovate in section, folded; penis cylindrical, slender, distal end tapered, unpigmented, with two lobes relatively distant positioned; basal lobe more developed and middle positioned. Distinguished from other closely related species by $>8.4 \%$ for COI.

\section{Etymology}

Referring to the second species of the genus Belgrandiellopsis gen. nov. discovered so far.

\section{Type material}

\section{Holotype}

TUNISIA - 1 spec. (sex unknown, preserved in ethanol 80\%); Bizerte Province, $40 \mathrm{~km}$ south of Mateur, Ettoute Spring; $36.883898^{\circ}$ N, 9.505894 E; 415 m a.s.1.; 15 Mar. 2015; N. Khalloufi leg.; MNCN 15.05/200098H.

\section{Paratypes}

TUNISIA • 5 specs (preserved in ethanol 80\%); same collection data as for holotype; MNCN 15.05/200098P - 26 specs (preserved in ethanol 80\%, 2 우, 2 재 dissected and 2 specs processed for DNA sequencing); same collection data as for holotype; UGSB 17666 - 250 specs (preserved in ethanol $95 \%$, ca 70 individuals dissected); same collection data as for holotype; Khalloufi's collection. 


\section{Type locality}

TUNISIA: Bizerte Province, $40 \mathrm{~km}$ south of Mateur, Ettoute Spring; $36.883898^{\circ} \mathrm{N}, 9.505894^{\circ} \mathrm{E}$; 415 m a.s.1.

\section{Description}

Measurements. Holotype: $\mathrm{SL}=2.09 \mathrm{~mm}, \mathrm{SW}=1.16 \mathrm{~mm}, \mathrm{SL} / \mathrm{SW}=1.80, \mathrm{AH}=0.90 \mathrm{~mm}$, SL-LBW $=$ $0.59 \mathrm{~mm}, \mathrm{WBW}=1.09 \mathrm{~mm}, \mathrm{AL}=0.99 \mathrm{~mm}, \mathrm{AW}=0.70 \mathrm{~mm}, \mathrm{WPW}=0.78 \mathrm{~mm}, \mathrm{WAW}=0.09 \mathrm{~mm}$.

SHELL. Elongate-conic, brown to yellowish, with 4-4.5 whorls (Fig. 5A-D; Table 2). Protoconch (Fig. 5G) obtuse, near planispiral, about 1.3 whorls, diameter about $350 \mu \mathrm{m}$; protoconch microsculpture granulated. Teleoconch whorls weakly convex, separated by deep sutures; body whorl occupying $2 / 3$ of total shell length. Aperture about $50 \%$ of total shell length, pyriform, strongly angled on the top and

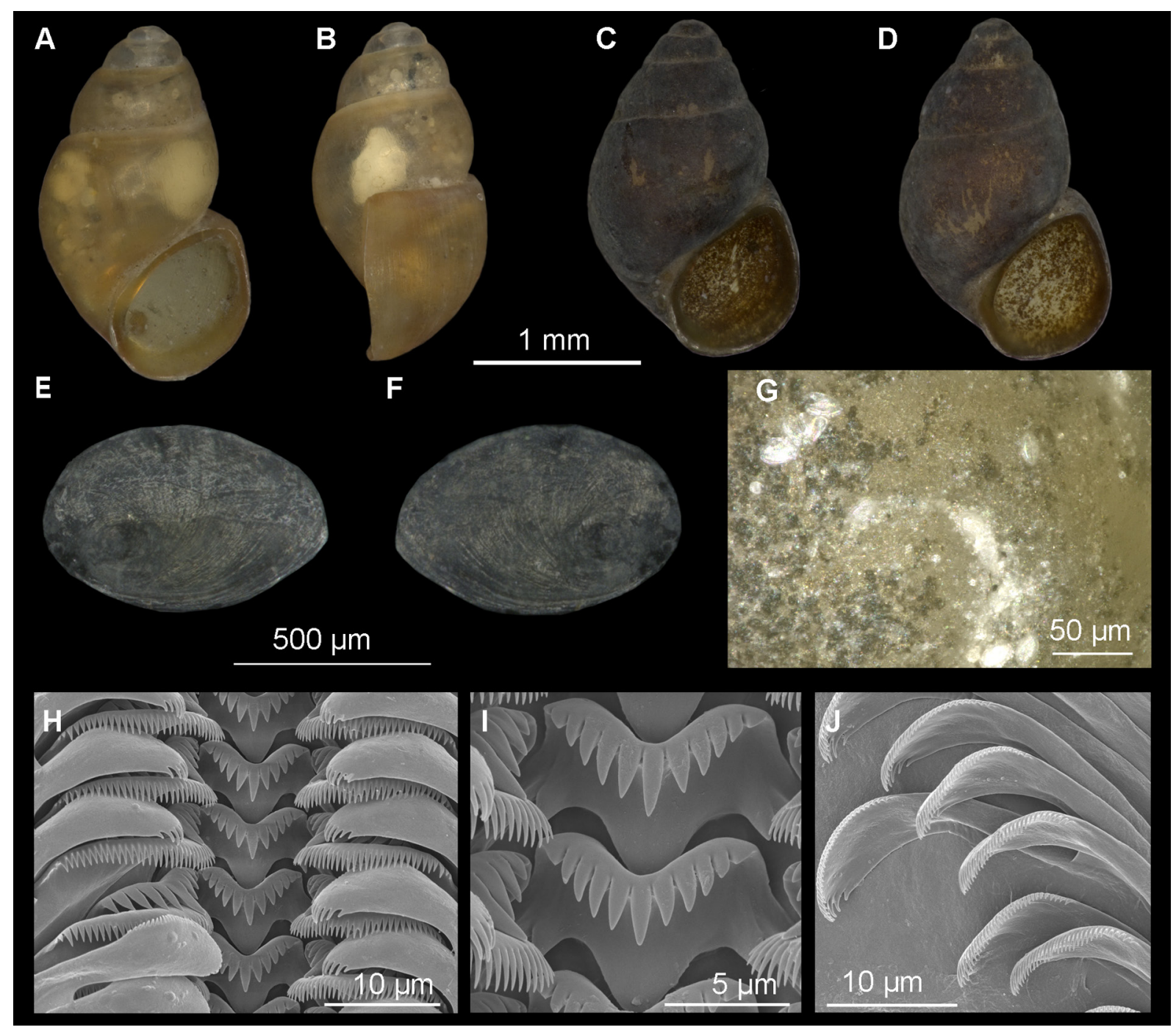

Fig. 5. Shells, opercula and radulae, Belgrandiellopsis secunda gen. et sp. nov. A-D. Shells. A-B. Holotype, MNCN 15.05/200098H. C-D. Paratypes, MNCN 15.05/200098P. E-F. Operculum $(\mathrm{E}=$ inner side; $\mathrm{F}=$ outer side). G. Detailed microsculpture of protoconch. $\mathbf{H}-\mathbf{J}$. Radulae. H. Portion of radula ribbon. I. Central radular teeth. J. Outer marginal teeth. 
narrowly disjunct from parietal wall of body whorl; inner lip thin but thicker than outer lip; peristome margin (Fig. 5B) straight. Umbilicus narrow and closed by inner lip.

Operculum. As for genus, thin, flat, whitish (nuclear region slightly yellowish), with ca 2 whorls (Fig. 5E-F); attachment scar area oval and located near nucleus.

RADULA. Length intermediate ( $25 \%$ total maximum shell length), having about 80 rows of teeth. Central tooth (Fig. 5H-I) formula (6)5-C-5(6)/1-1, cutting edge slightly concave; basal tooth face M-shaped and relatively narrow wings; basal tongue broadly V-shaped, about equal to lateral margins; a single small pair of basal cusps, triangular. Lateral teeth (Fig. 5H) formula 5-C-3(4); central cusp large, narrow, pointed. Inner marginal teeth having 32-35 sharp cusps. Outer marginal teeth having 30-33 sharp cusps (Fig. 5J).

Pigmentation and anatomy. Visceral coil pale-yellowish, showing by transparency the gonad. Pallial coil with uniform and dense brown pigmentation on roof (left side), anterior edge of mantle, foot and between tentacles (Fig. 6C). Black small eyes surrounded by brownish pigment. Snout with medium distal lobation. Tentacles parallel-side, distal end nonexpanded. Ctenidium filaments about 12, small, taller than wide. Osphradium whitish, ellipsoidal, positioned centrally along ctenidium (Fig. 6A). Stomach slightly longer than wide with a small gastric (posterior) caecum (Fig. 6B); rectum wide, folded and S-shaped.

Male genitalia. Penis cylindrical (Fig. 6D), medium sized, unpigmented, slender, slightly folded; filament short, tapering; basal portion intermediate in width, longer than distal portion, attached centrally to the head well behind the eyes; penis having two developed lobes, both with distal papilla, showing by transparency tubular glands; lobes located relatively distant from one another on opposite sides of penis; basal lobe large, conic, sessile, positioned centrally along penis; distal lobe smaller, pyriform, pedunculated. Prostate gland small, close, bean-shaped, longer than wide $(0.2 \mathrm{~mm} / 0.10 \mathrm{~mm})$. Posterior vas deferens whitish, forming the seminal vesicle and developed in its median portion with several loops (about $3.5 \mathrm{~mm}$ in length and $0.05 \mathrm{~mm}$ in section). Pallial vas deferens straight, narrow, whitish, unciliated, entering the posteroventral portion of gland and exiting from its anterior end.

Female Genitalia. Ovary simple, with stalked lobes, occupying near $40 \%$ of visceral coil. Distal female genitalia occupying near $50 \%$ of pallial roof; albumen gland less developed than capsule gland (Fig. 6E). Bursa copulatrix ovoid to pyriform, having a duct slightly shorter than bursal length. Renal oviduct unpigmented, coiled, having a very small, ovoid to spherical pouch at the end of the coiled section. Two seminal receptacles; SR1 medium sized, elongate, pedunculate, joining renal oviduct just above the insertion point with bursal duct; SR2 small, globular, sessile, located on renal oviduct near loop before pouch (Fig. 6F-G).

\section{Ecology and distribution}

This species was found in a spring and its outflow in the rural village Ettoute between the cities of Mateur and Beja, in northern Tunisia. Specimens were attached to the underside of gravel and stones covered by algae. In the small stream flowing out of the spring, the snails were found in the central section featuring clean and oxygenated waters and a silty substrate. The density of this species decreased drastically downstream from the water resurgence. Co-occurring gastropod species were Pseudamnicola sp., Galba truncatula (O.F. Müller, 1774), Bulinus truncatus (Audouin, 1827) and Physella acuta Draparnaud, 1805.

\section{Remarks}

Despite similarities in mean COI divergence between this species and the closely related species Bullaregia tunisiensis Khalloufi, Béjaoui \& Delicado, 2017, Biserta putealis gen. et sp. nov. and 
Belgrandiellopsis chorfensis gen. et sp. nov. (9.1\%, 8.9\% and 8.4\%, respectively), B. secunda gen. et sp. nov. was assigned to Belgrandiellopsis gen. nov. based on the combination of two glandular lobes located relatively distant from one another on opposite sides of the penis [one lobe in Bullaregia (Khalloufi et al. 2017), two lobes closely positioned to one another in Biserta gen. nov.], small bursa copulatrix with a long duct (larger bursa copulatrix with a shorter duct in Biserta gen. nov.), small gastric caecum (larger in Bullaregia) and small shell umbilicus (larger in Biserta gen. nov.).

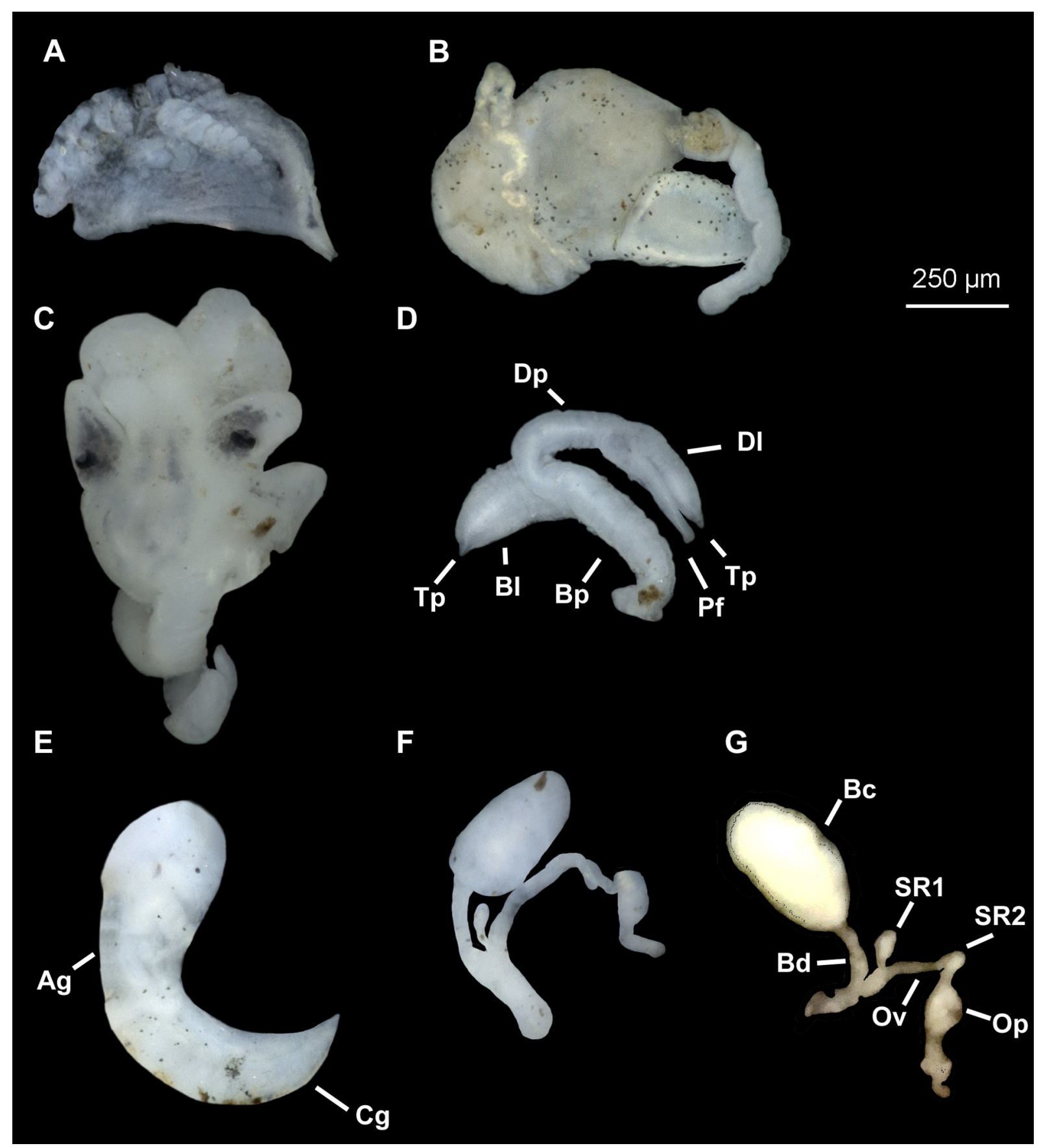

Fig. 6. Anatomy of Belgrandiellopsis secunda gen. et sp. nov. (paratypes, UGSB 17666). A. Ctenidium and osphradium. B. Stomach. C. Head of male and penis. D. Detail of penis and penis lobes. E. Pallial oviduct. F. Bursa copulatrix and seminal receptacles. G. Ditto, observed by transmitted light. Anatomical abbreviations explained in the text. 
Genus Biserta gen. nov.

urn:1sid:zoobank.org:act:11183287-E4AD-4FBB-A304-E7BB699133AA

\section{Type species}

Biserta putealis sp. nov., by present designation.

\section{Diagnosis}

Shell small (maximum length $2 \mathrm{~mm}$ ), ovate-conic to elongate-conic, with obtuse and planispiral protoconch; whorls convex, with deep sutures. Penultimate whorl tall relative to remaining whorls. Aperture pyriform to ovate, complete, slightly angled on top and often fused to the body whorl. Umbilicus closed by inner lip. Operculum corneous, whitish to yellowish, thin, pliable, elongate-ellipsoidal, spiral, paucispiral, with submarginal nucleus. One pair of small triangular basal cusps on radular central tooth. Ctenidium well developed. Presence of bursa copulatrix; unpigmented renal oviduct with a spherical pouch at the end of the coiled section; two seminal receptacles. Penis attached to the neck behind the right eye with two glandular lobes closely positioned to one another, each of them bearing a terminal papilla.

\section{Etymology}

Biserta is one of the Latin names of Bizerte, name of the province where the genus was discovered; gender feminine.

\section{Remarks}

Biserta gen. nov. differs from the closely related genera Bullaregia and Belgrandiellopsis gen. nov. according to its larger shell umbilicus, two glandular lobes closely positioned to one another on the penis, slightly larger bursa copulatrix, shorter bursal duct and to its $8-8.9 \%$ COI sequence divergence.

Biserta putealis gen. et sp. nov.

urn:1sid:zoobank.org:act:AB67B9A3-8BA6-4AC2-97E3-97C61E6A4C32

Figs $7-8$

\section{Diagnosis}

Shell 1.6 to $2.0 \mathrm{~mm}$ high, elongate-conic, brown-yellowish; central radular tooth formula (5)4-C4(5)/1-1; bursa copulatrix pyriform, with a short duct; two seminal receptacles, SR1 more developed and pedunculated, SR2 small, globular, located near loop before the pouch; prostate gland bean shaped, ovate in section, folded; penis gradually tapering, unpigmented, with two sessile lobes closely positioned to one another. Distinguished from other closely related species by $>8.0 \%$ for COI.

\section{Etymology}

The species epithet is from the Latin adjective 'putealis', meaning 'from a well'.

\section{Type material}

Holotype

TUNISIA 11 spec. (sex unknown, preserved in ethanol 80\%); Bizerte Province, $7 \mathrm{~km}$ south of Menzel Bourguiba, Soudene Well; 37.09046 N, 9.777997 E; 115 m a.s.1.; 15 Mar. 2015; N. Khalloufi leg.; MNCN 15.05/200099H.

Paratypes

TUNISIA - 5 specs (preserved in ethanol 80\%); same collection data as for holotype;

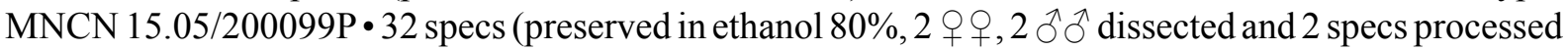


for DNA sequencing); same collection data as for holotype; UGSB $17664 \cdot 70$ specs (preserved in ethanol 95\%, ca 40 individuals dissected); same collection data as for holotype; Khalloufi's collection.

\section{Type locality}

TUNISIA: Bizerte Province, 7 km south of Menzel Bourguiba, Soudene Well; $37.09046^{\circ} \mathrm{N}, 9.777997^{\circ} \mathrm{E}$; 115 m a.s.l.

\section{Description}

MEASUREMENTs. Holotype: $\mathrm{SL}=2.00 \mathrm{~mm}, \mathrm{SW}=1.04 \mathrm{~mm}, \mathrm{SL} / \mathrm{SW}=1.92, \mathrm{AH}=0.98 \mathrm{~mm}$, SL-LBW $=$ $0.70 \mathrm{~mm}, \mathrm{WBW}=0.97 \mathrm{~mm}, \mathrm{AL}=0.93 \mathrm{~mm}, \mathrm{AW}=0.70 \mathrm{~mm}, \mathrm{WPW}=0.78 \mathrm{~mm}, \mathrm{WAW}=0.17 \mathrm{~mm}$.

Shell. Elongate-conic, brown to yellowish, with 4-4.5 whorls (Fig. 7A-D; Table 2). Protoconch (Fig. 7G) obtuse, near planispiral, about 1.25 whorls, diameter about $300 \mu \mathrm{m}$; protoconch microsculpture

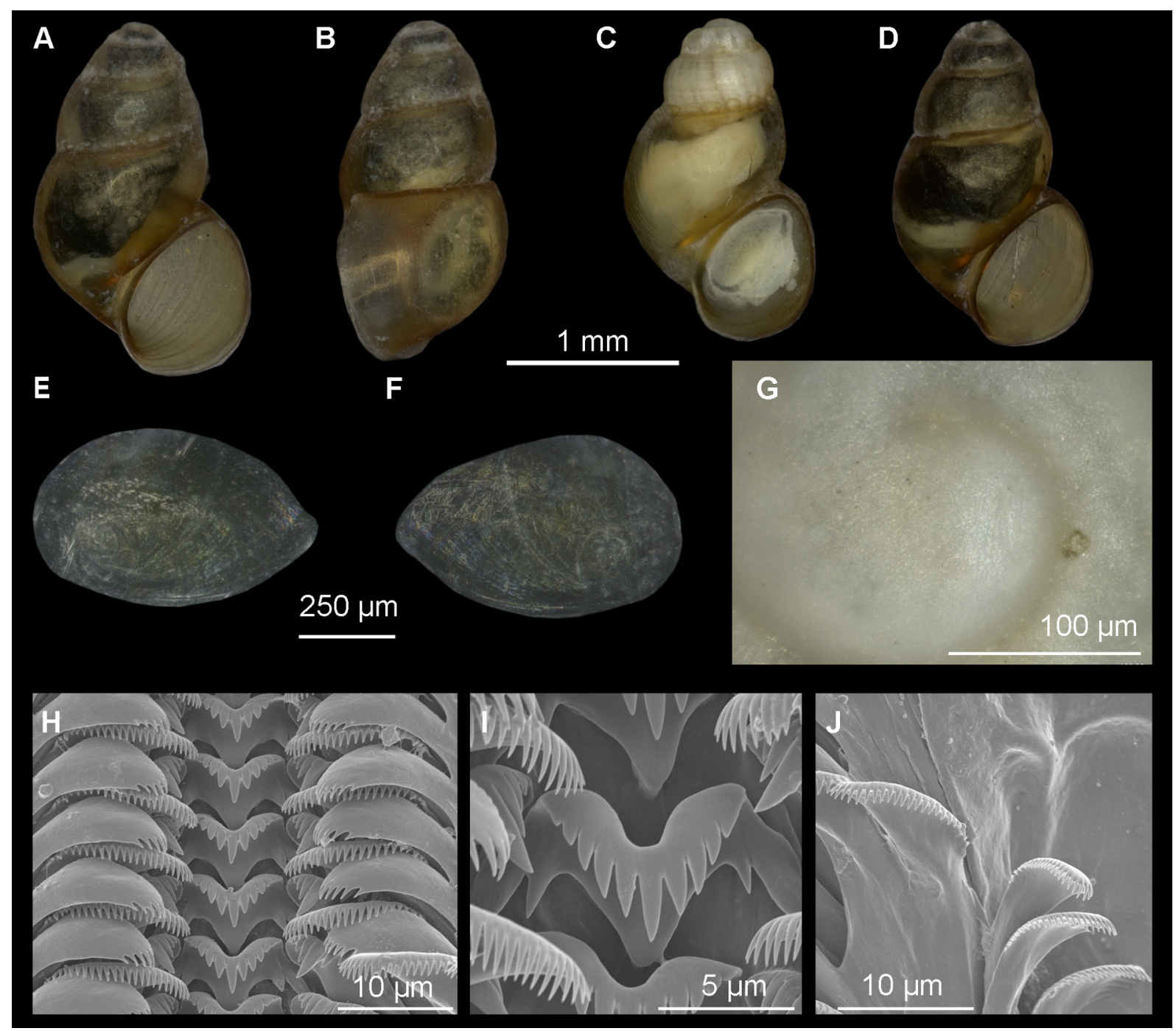

Fig. 7. Shells, opercula and radulae, Biserta putealis gen. et sp. nov. A-D. Shells. A-B. Holotype, MNCN 15.05/200099H. C-D. Paratypes, MNCN 15.05/200099P. E-F. Operculum (E = inner side; $\mathrm{F}=$ outer side). G. Detailed microsculpture of protoconch. H-J. Radulae. H. Portion of radula ribbon. I. Central radular teeth. J. Marginal teeth. 
granulated. Teleoconch whorls convex, separated by deep sutures; body whorl occupying $2 / 3$ of total shell length. Aperture about $50 \%$ of total shell length, pyriform, slightly angled on the top and narrowly disjunct from parietal wall of body whorl; inner lip thin but thicker than outer lip; peristome margin (Fig. 7B) straight. Umbilicus narrow and closed by inner lip.

Operculum. As for genus, thin, flat, whitish (nuclear region slightly yellowish), with ca 2 whorls (Fig. 7E-F); attachment scar area oval and located near nucleus.

RADULA. Length intermediate ( $25 \%$ total maximum shell length), having about 80 rows of teeth. Central tooth (Fig. 7H-I) formula (5)4-C-4(5)/1-1, cutting edge strongly concave; basal tooth face M-shaped and relatively narrow wings; basal tongue broadly $\mathrm{V}$-shaped, about equal to lateral margins; a single small pair of basal cusps, triangular. Lateral teeth (Fig. 7H) formula (4)3-C-5(6); central cusp large, narrow, pointed. Inner marginal teeth having 23-25 sharp cusps. Outer marginal teeth having 22-23 sharp cusps (Fig. 7J).

Pigmentation And ANATOMy. Visceral coil brown-yellowish, showing by transparency the gonad; pallial coil with uniform and dense pigmentation on the roof, anteriorly to edge of mantle, foot and area between tentacles (Fig. 8C). Black small eyes surrounded by brownish pigment. Snout with medium distal lobation. Tentacles parallel-side, distal end nonexpanded. Ctenidium filaments about 13, small, taller than wide. Osphradium whitish, ellipsoidal, positioned centrally along ctenidium (Fig. 8A). Stomach slightly longer than wide with a small gastric (posterior) caecum (Fig. 8B); rectum wide, folded and S-shaped.

MaLe Genitalia. Penis gradually tapering (Fig. 8D), medium sized, unpigmented, slightly folded; filament short, tapering; basal portion intermediate in width, shorter than distal portion, attached centrally to the head well behind the eyes; penis having two developed lobes, very close to one another on opposite sides of penis; both lobes having distal papilla and showing by transparency tubular glands; ventral lobe large, pyriform, pedunculated; dorsal lobe small, pyriform, sessile. Prostate gland small, close, beanshaped, longer than wide $(0.25 \mathrm{~mm} / 0.05 \mathrm{~mm})$. Posterior vas deferens with several loops, yellowish and developed in its median portion (about $2 \mathrm{~mm}$ in length and $0.05 \mathrm{~mm}$ in section). Pallial vas deferens straight, narrow, whitish, unciliated, entering the posteroventral portion of gland and exiting from its anterior end.

Female genitalia. Ovary simple, with stalked lobes, occupying near $40 \%$ of visceral coil. Distal female genitalia occupying near $50 \%$ of pallial roof; albumen gland approximately as long as capsule gland (Fig. 8E). Bursa copulatrix pyriform, having a shorter duct than bursal length. Renal oviduct unpigmented, coiled, having a spherical pouch at the end of the coiled section. Two seminal receptacles; SR1 medium sized, elongate, pedunculate, joining renal oviduct just above the insertion point with bursal duct; SR2 small, globular, sessile, located on renal oviduct near loop before pouch (Fig. 8F-G).

\section{Ecology and distribution}

This species was found only in a small well in southern Menzel Bourguiba city, northern Tunisia. The well is $1.5 \mathrm{~m}$ in diameter and $4 \mathrm{~m}$ deep. Specimens were very abundant on the rock walls and on the rocky and sandy bottom. Water salinity was about 1.5 ppt. Pseudamnicola sp. was the only co-occurring gastropod species.

\section{Remarks}

Some individuals of the collected population lack body pigmentation (Fig. 7C), which could indicate adaptation to subterranean conditions (Delicado 2018). Other anatomical structures resembled those of the pigmented individuals. 


\section{Discussion}

The discovery of Bullaregia tunisiensis by Khalloufi et al. (2017) prompted our intense research on groundwater snails in an effort to gain a better understanding of the species richness, distribution and evolutionary patterns of hydrobiid clades in Tunisia. In prior work (Khalloufi et al. 2017), we detected an uncertain phylogenetic position of this genus within the Hydrobiidae. In the present study, we provide morphological and genetic data for previously unknown populations from Tunisian groundwater systems and evidence of three new taxa resolved in a monophyletic group together with $B$. tunisiensis. In our phylogenetic analyses (Fig. 2), no well-supported relationship was found between this group and the recently discovered clade of subterranean hydrobiid species from Bulgaria

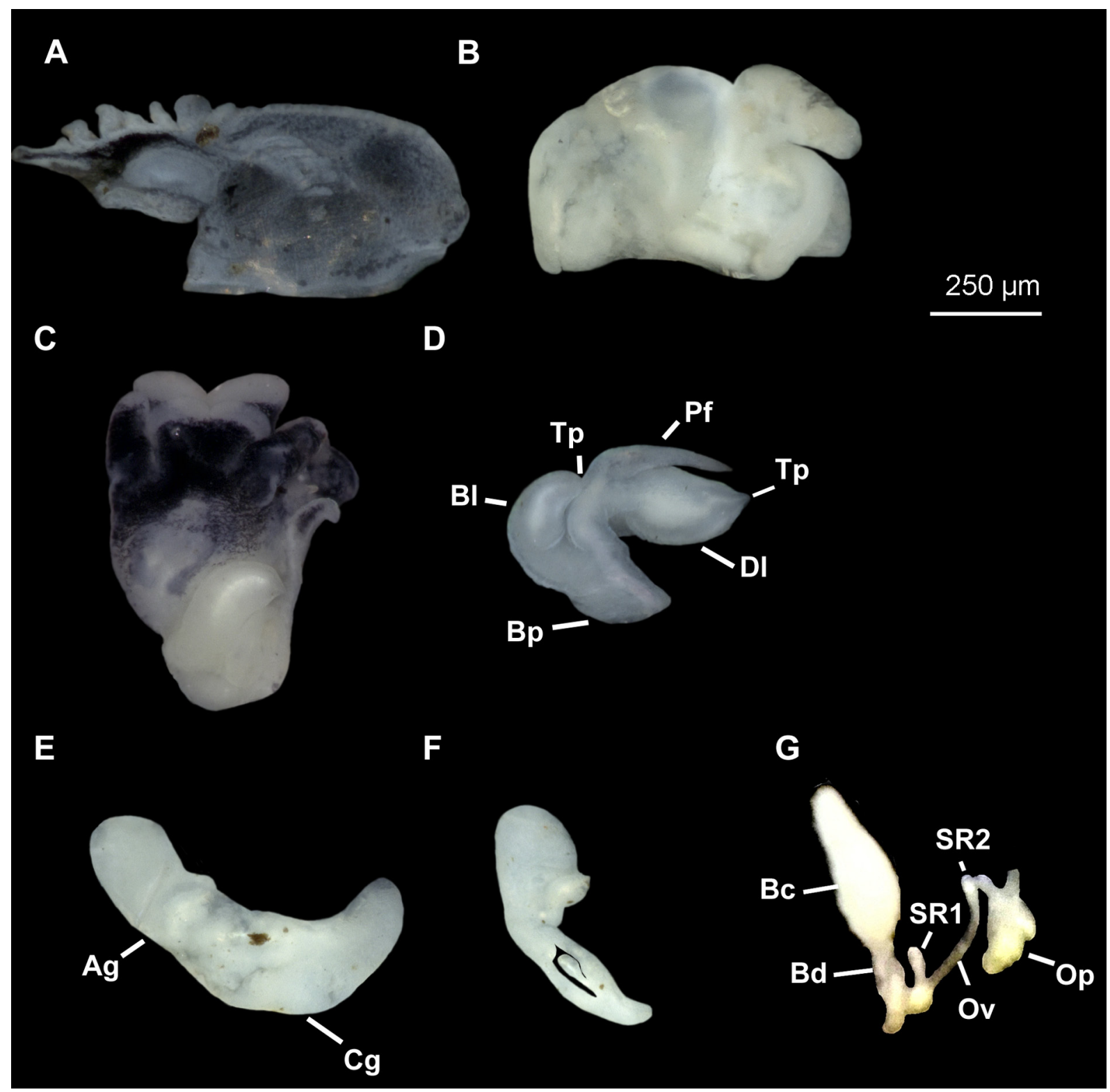

Fig. 8. Anatomy of Biserta putealis gen. et sp. nov. (paratypes, UGSB 17664). A. Ctenidium and osphradium. B. Stomach. C. Head of male and penis. D. Detail of penis and penis lobes. E. Pallial oviduct. F. Bursa copulatrix and seminal receptacles. G. Ditto, observed by transmitted light. Anatomical abbreviations explained in the text. 
(Osikowski et al. 2017); instead, it formed a monophyletic group with Corbellaria celtiberica and Kerkia kusceri. The sister relationship between these two species of valvatiform snails was previously shown by Delicado et al. (2019). However, this grouping was not supported by morphological evidence as, for instance, the Tunisian snails feature elongate-conic shells and remarkable differences in female and male genitalia (see Girardi \& Boeters 2012 and Bodon et al. 2001 for morphological comparison with $C$. celtiberica and $K$. kusceri, respectively). We anticipate that additional genetic data from undiscovered populations of groundwater and spring snails living in unexplored areas such as Tunisia will change this phylogenetic picture and help to further unravel the phylogenetic position of this Tunisian clade within the Hydrobiidae.

The two new genera presented in this study raises the number of genera of Hydrobiidae sensu stricto in Tunisia to seven (Pseudamnicola, Hydrobia, Ecrobia Stimpson, 1865, Mercuria, Bullaregia, Belgrandiellopsis gen. nov., Biserta gen. nov.; Wilke et al. 2002; Glöer et al. 2010; Delicado et al. 2015; Khalloufi et al. 2017; Vandendorpe et al., 2019) and to eight for North Africa (García et al. 2010). The three subterranean genera recovered here as a monophyletic group (i.e., the Tunisian clade) are as genetically divergent (8.0-9.2\% COI p-distances) as within other clades of hydrobiid subterranean genera (e.g., 7.8-11.8\% COI p-distances; Osikowski et al. 2017). Although this genetic divergence falls into the range of intrageneric genetic variation reported for spring genera of Hydrobiidae (e.g., 5.3911.15\% COI p-distances within Corrosella Boeters, 1970; Delicado et al. 2012), we found sufficient morphological evidence to assign the three collected populations to two distinct new genera outside Bullaregia. The three genera comprising the Tunisian clade (i.e., Bullaregia, Belgrandiellopsis gen. nov. and Biserta gen. nov.) differ mainly in anatomical rather than in shell shape. For instance, Bullaregia presents a single medial lobe on the outer edge of the penis (two lobes on both edges of the penis in Belgrandiellopsis gen. nov. and Biserta gen. nov., more distally positioned in the former genus) and more cusps on the radular teeth.

These new genera and species show a limited distribution in Tunisia, as they are endemic to one or two localities. However, their distribution could extend to other localities in northern Tunisia and northeastern Algeria. Considering that many groundwater bodies have not yet been explored, the number of endemic taxa in Tunisia is likely larger. Thus, field surveys in these aquatic systems should be intensified to discover and inventory their faunal diversity. This point is crucial for the design of conservation measures before these local endemic species become extinct.

\section{Acknowledgements}

We thank B. Hoenig and S. Agel (Imaging Unit, Biomedical Research Centre Seltersberg, Justus Liebig University, Giessen) for their assistance with the ESEM photomicrographs. The English was improved by Ana Burton. We thank the editor and two anonymous reviewers for their valuable and constructive comments. This work was funded by a German Science Foundation (DFG) grant (DE 2605/1-1) to D. Delicado.

\section{References}

Akaike H. 1974. A new look at the statistical model identification. IEEE Transactions on Automatic Control 19 (6): 716-723. https://doi.org/10.1109/TAC.1974.1100705

Bodon M., Manganelli G. \& Giusti F. 2001. A survey of the European valvatiform hydrobiid genera with special reference to Hauffenia Pollonera, 1898 (Gastropoda: Hydrobiidae). Malacologia 43: 103-215.

Boeters H.D. 1976. Hydrobiidae Tunesiens. Archiv für Molluskenkunde 107 (1-3): 89-105.

Bourguignat J.R. 1860. Malacologie terrestre et fluviatile de la Bretagne. J.B. Baillière, Paris. https://doi.org/10.5962/bhl.title.10634 
KHALLOUFI N. et al., New hydrobiid genera and species from Tunisia

Bourguignat J.R. 1864. Malacologie de l'Algérie ou histoire naturelle des animaux mollusqes terrestres et fluviatiles: recueillis jusqu'à ce jour dans nos possessions du nord de l'Afrique. Challamel Ainé, Paris.

Culver D.C. 2012. Mollusks. In: White W. \& Culver D. (eds) Encyclopedia of Caves. $2^{\text {nd }}$ Ed.: 512-517. Academic Press, Amsterdam. https://doi.org/10.1016/B978-0-12-383832-2.00074-8

Darriba D., Taboada G.L., Doallo R. \& Posada D. 2012. jModelTest 2: more models, new heuristics and parallel computing. Nature Methods 9 (8): 772-772. https://doi.org/10.1038/nmeth.2109

Davis G.M., Wilke T., Spolsky C., Qiu C.-P., Qiu D.-C., Xia M.-Y., Zhang Y. \& Rosenberg G. 1998. Cytochrome oxidase I-based phylogenetic relationships among the Pomatiopsidae, Hydrobiidae, Rissoidae and Truncatellidae (Gastropoda: Caenogastropoda: Rissoacea). Malacologia 40: 251-266.

Debeaux O. 1863. Notice sur quelques Mollusques nouveaux, ou peu connus, de la grande Kabylie. Journal de Conchyliologie 11: 10-21.

Delicado D. 2018. A rare case of stygophily in the Hydrobiidae (Gastropoda: Sadleriana). Journal of Molluscan Studies 84 (4): 480-485. https://doi.org/10.1093/mollus/eyy032

Delicado D., Machordom A. \& Ramos M.A. 2012. Underestimated diversity of hydrobiid snails. The case of Pseudamnicola (Corrosella) (Mollusca: Caenogastropoda: Hydrobiidae). Journal of Natural History 46 (1-2): 25-89. https://doi.org/10.1080/00222933.2011.623358

Delicado D., Machordom A. \& Ramos M.A. 2015. Effects of habitat transition on the evolutionary patterns of the microgastropod genus Pseudamnicola (Mollusca, Hydrobiidae). Zoologica Scripta 44 (4): 403-417. https://doi.org/10.1111/zsc.12104

Delicado D., Hauffe T. \& Wilke T. 2018. Ecological opportunity may facilitate diversification in Palearctic freshwater organisms: a case study on hydrobiid gastropods. BMC Evolutionary Biology 18 (1): 55. https://doi.org/10.1186/s12862-018-1169-2

Delicado D., Arconada B., Aguado A. \& Ramos M.A. 2019. Multilocus phylogeny, species delimitation and biogeography of Iberian valvatiform springsnails (Caenogastropoda: Hydrobiidae), with the description of a new genus. Zoological Journal of the Linnean Society 186 (4): 892-914.

https://doi.org/10.1093/zoolinnean/zly093

Diehl E., Jauker B., Albrecht C., Wilke T. \& Wolters V. 2018. GIEßEN: University Collections: Justus Liebig University Gießen. In: Beck L.A. (ed.) Zoological Collections of Germany. The Animal Kingdom in its Amazing Plenty at Museums and Universities: 373-381. Springer, Cham.

Falniowski A. \& Szarowska M. 2011. A new genus and new species of valvatiform hydrobiid (Rissooidea; Caenogastropoda) from Greece. Molluscan Research 31 (3): 189-199.

Falniowski A. \& Szarowska M. 2012. Phylogenetic position of Boleana umbilicata (Kuščer, 1932) (Caenogastropoda: Rissooidea). Folia Malacologica 20 (4): 265-270.

https://doi.org/10.2478/v10125-012-0022-2

Falniowski A. \& Szarowska M. 2013. Phylogenetic relationships of Dalmatinella fluviatilis Radoman, 1973 (Caenogastropoda: Rissooidea). Folia Malacologica 21 (1): 1-7.

https://doi.org/10.12657/folmal.021.001

Folmer O., Black M., Hoeh W., Lutz R. \& Vrijenhoek R. 1994. DNA primers for amplification of mitochondrial cytochrome $c$ oxidase subunit I from diverse metazoan invertebrates. Molecular Marine Biology and Biotechnology 3 (5): 294-299.

Fox J. \& Weisberg S. 2019. An R Companion to Applied Regression, $3^{\text {rd }}$ Ed. Sage, Thousand Oaks, CA. García N., Cuttelod A. \& Malak D.A. 2010. The Status and Distribution of Freshwater Biodiversity in Northern Africa. IUCN, Gland, Switzerland, Cambridge, UK and Malaga, Spain. Available from 
https://www.iucn.org/es/content/status-and-distribution-freshwater-biodiversity-northern-africa-1 [accessed 21 Apr. 2020].

Gibert J. \& Culver D.C. 2009. Assessing and conserving groundwater biodiversity: an introduction. Freshwater Biology 54 (4): 639-648. https://doi.org/10.1111/j.1365-2427.2009.02202.x

Girardi H. \& Boeters H.D. 2012. Corbellaria celtiberica gen. et sp. nov. (Gastropoda: Hydrobiidae), mollusque valvatiforme stygobie de la province de Soria (Péninsule Ibérique). Spira 4 (3-4): 149-160.

Glöer P., Bouzid S. \& Boeters H.D. 2010. Revision of the genera Pseudamnicola Paulucci 1878 and Mercuria Boeters 1971 from Algeria with particular emphasis on museum collections (Gastropoda: Prosobranchia: Hydrobiidae). Archiv für Molluskenkunde 139 (1): 1-22.

https://doi.org/10.1127/arch.moll/1869-0963/139/001-022

Hershler R. \& Ponder W.F. 1998. A review of morphological characters of hydrobioid snails. Smithsonian Contributions to Zoology 600: 1-55. https://doi.org/10.5479/si.00810282.600

Hershler R., Liu H.-P. \& Howard J. 2014. Springsnails: a new conservation focus in western North America. BioScience 64: 693-700. https://doi.org/10.1093/biosci/biu100

Holland P.W.H., Hacker A.M. \& Williams N.A. 1991. A molecular analysis of the phylogenetic affinities of Saccoglossus cambrensis Brambell \& Cole (Hemichordata). Philosophical Transactions of the Royal Society B. Biological Sciences 332 (1264): 185-189. https://doi.org/10.1098/rstb.1991.0048

Hurvich C.M. \& Tsai C.-L. 1989. Regression and time series model selection in small samples. Biometrika 76 (2): 297-307. https://doi.org/10.1093/biomet/76.2.297

Issel A. 1880. Molluschi terrestri e d'acqua dolce viventi e fossili della Tunisia. Tipografia del R. Istituto sordo-muti, Genoa.

Katoh K., Misawa K., Kuma K. \& Miyata T. 2002. MAFFT: a novel method for rapid multiple sequence alignment based on fast Fourier transform. Nucleic Acids Research 30 (14): 3059-3066.

Khalloufi N. \& Boumaiza M. 2007. Première citation et description de quatre pulmonés dulcicoles de Tunisie (Mollusca, Orthogastropoda). Bulletin de la Société zoologique de France 132 (3): 191-204.

Khalloufi N., Béjaoui M. \& Delicado D. 2017. A new genus and species of uncertain phylogenetic position within the family Hydrobiidae (Caenogastropoda: Truncatelloidea) discovered in Tunisian springs. European Journal of Taxonomy 328: 1-15. https://doi.org/10.5852/ejt.2017.328

Kimura M. 1981. Estimation of evolutionary distances between homologous nucleotide sequences. Proceedings of the National Academy of Sciences of the USA 78 (1): 454-458.

https://doi.org/10.1073/pnas.78.1.454

Kumar S., Stecher G. \& Tamura K. 2016. MEGA7: Molecular Evolutionary Genetics Analysis version 7.0 for bigger datasets. Molecular Biology and Evolution 33 (7): 1870-1874.

https://doi.org/10.1093/molbev/msw054

Letourneux A. \& Bourguignat J.R. 1887. Prodrome de la Malacologie terrestre et fluviatile de la Tunisie. Imprimerie nationale, Paris. https://doi.org/10.5962/bhl.title.132280

Miller J.P., Ramos M.A., Hauffe T. \& Delicado D. 2018. Global species richness of hydrobiid snails determined by climate and evolutionary history. Freshwater Biology 63 (10): 1225-1239.

https://doi.org/10.1111/fwb.13128

Osikowski A., Hofman S., Georgiev D., Rysiewska A. \& Falniowski A. 2017. Unique, ancient stygobiont clade of Hydrobiidae (Truncatelloidea) in Bulgaria: the origin of cave fauna. Folia Biologica 65 (2): 79-93. https://doi.org/10.3409/fb65_2.79 
KHALLOUFI N. et al., New hydrobiid genera and species from Tunisia

Ouedraogo I., Defourny P. \& Vanclooster M. 2016. Mapping the groundwater vulnerability for pollution at the pan African scale. Science of The Total Environment 544: 939-953.

https://doi.org/10.1016/j.scitotenv.2015.11.135

Pallary P. 1921. Faune malacologique du grand Atlas. Journal de Conchyliologie 66: 81-217.

Pallary P. 1923. Faune malacologique des eaux douces de la Tunisie. Archives des Instituts Pasteur de l'Afrique du nord 12: 22-47.

Rambaut A. 2010. FigTree. Available from http://tree.bio.ed.ac.uk/software/figtree [accessed 19 Apr. 2020].

R Development Core Team 2019. R: A language and environment for statistical computing. R Foundation for Statistical Computing, Vienna, Austria.

Ronquist F., Teslenko M., van der Mark P., Ayres D.L., Darling A., Höhna S., Larget B., Liu L., Suchard M.A. \& Huelsenbeck J.P. 2012. MrBayes 3.2: Efficient Bayesian phylogenetic inference and model choice across a large model space. Systematic Biology 61 (3): 539-542.

https://doi.org/10.1093/sysbio/sys029

Rysiewska A., Georgiev D., Osikowski A., Hofman S. \& Falniowski A. 2016. Pontobelgrandiella Radoman, 1973 (Caenogastropoda : Hydrobiidae) : a recent invader of subterranean waters. Journal of Conchology 42 (4): 193-203.

Rysiewska A., Prevorčnik S., Osikowski A., Hofman S., Beran L. \& Falniowski A. 2017. Phylogenetic relationships in Kerkia and introgression between Hauffenia and Kerkia (Caenogastropoda: Hydrobiidae). Journal of Zoological Systematics and Evolutionary Research 55 (2): 106-117.

https://doi.org/10.1111/jzs.12159

Schreiber K., Hauffe T., Albrecht C. \& Wilke T. 2012. The role of barriers and gradients in differentiation processes of pyrgulinid microgastropods of Lake Ohrid. Hydrobiologia 682 (1): 61-73.

https://doi.org/10.1007/s10750-011-0864-4

Seurat L.G. 1921. Faune des Eaux continentales de la Berbérie. Travaux du Laboratoire de Zoologie Appliquée, Faculté des Sciences, Université d'Alger, Algeria.

Seurat L.G. 1934. Formations littorales et estuaires de la Syrte Mineure (Golfe de Gabés). Bulletin de la Station océanographique de Salammbô 32: 1-65.

Stamatakis A. 2014. RAxML version 8: a tool for phylogenetic analysis and post-analysis of large phylogenies. Bioinformatics 30 (9): 1312-1313. https://doi.org/10.1093/bioinformatics/btu033

Strong E.E., Gargominy O., Ponder W.F. \& Bouchet P. 2008. Global diversity of gastropods (Gastropoda; Mollusca) in freshwater. In: Balian E.V., Lévêque C., Segers H. \& Martens K. (eds) Freshwater Animal Diversity Assessment: 149-166. Springer, Netherlands. https://doi.org/10.1007/s10750-007-9012-6

Sugiura N. 1978. Further analysts of the data by Akaike's information criterion and the finite corrections. Communications in Statistics - Theory and Methods 7 (1): 13-26.

https://doi.org/10.1080/03610927808827599

Szarowska M. 2006. Molecular phylogeny, systematics and morphological character evolution in the Balkan Rissooidea (Caenogastropoda). Folia Malacologica 14 (3): 99-168.

https://doi.org/10.12657/folmal.014.014

Szarowska M. \& Falniowski A. 2011. An unusual, flagellum-bearing hydrobiid snail (Gastropoda: Rissooidea: Hydrobiidae) from Greece, with descriptions of a new genus and a new species. Journal of Natural History 45 (35-36): 2231-2246. https://doi.org/10.1080/00222933.2011.591067 
Szarowska M. \& Falniowski A. 2014. Horatia Bourguignat, 1887: is this genus really phylogenetically very close to Radomaniola Szarowska, 2006 (Caenogastropoda: Truncatelloidea)? Folia Malacologica 22 (1): 31-39. https://doi.org/10.12657/folmal.022.003

Szarowska M., Grzmil P., Falniowski A. \& Sirbu I.O. 2007. Grossuana codreanui (Grossu, 1946) and the phylogenetic relationships of the East Balkan genus Grossuana (Radoman, 1973) (Gastropoda: Rissooidea). Hydrobiologia 579: 379-391. https://doi.org/10.1007/s10750-006-0530-4

Tamura K. \& Nei M. 1993. Estimation of the number of nucleotide substitutions in the control region of mitochondrial DNA in humans and chimpanzees. Molecular Biology and Evolution 10 (3): 512-526. https://doi.org/10.1093/oxfordjournals.molbev.a040023

Tavaré S. 1986. Some probabilistic and statistical problems in the analysis of DNA sequences. American Mathematical Society: Lectures on Mathematics in the Life Sciences 17: 57-86.

Terver A.P. 1839. Catalogue des mollusques terrestres et fluviatiles, observés dans les possessions françaises, au nord de l'Afrique. J.B. Baillière, Paris and Lyon, France.

Vandendorpe J., van Baak C.G.C., Stelbrink B., Delicado D., Albrecht C. \& Wilke T. 2019. Historical faunal exchange between the Pontocaspian Basin and North America. Ecology and Evolution 9 (18): 10816-10827. https://doi.org/10.1002/ece3.5602

Wilke T., Davis G.M., Gong X. \& Liu H.X. 2000. Erhaia (Gastropoda: Rissooidea): phylogenetic relationships and the question of Paragonimus coevolution in Asia. The American Journal of Tropical Medicine and Hygiene 62 (4): 453-459. https://doi.org/10.4269/ajtmh.2000.62.453

Wilke T., Davis G.M., Falniowski A., Giusti F., Bodon M. \& Szarowska M. 2001. Molecular systematics of Hydrobiidae (Mollusca: Gastropoda: Rissooidea): testing monophyly and phylogenetic relationships. Proceedings of the Academy of Natural Sciences of Philadelphia 151 (1): 1-21.

https://doi.org/10.1635/0097-3157(2001)151[0001:MSOHMG]2.0.CO;2

Wilke T., Pfenninger M. \& Davis G.M. 2002. Anatomical variation in cryptic mudsnail species: statistical discrimination and evolutionary significance. Proceedings of the National Academy of Sciences of Philadelphia 152: 45-66. https://doi.org/10.1635/0097-3157(2002)152[0045:avicms]2.0.co;2

Wilke T., Davis G.M., Qiu D.C. \& Spear R.C. 2006. Extreme mitochondrial sequence diversity in the intermediate schistosomiasis host Oncomelania hupensis robertsoni: another case of ancestral polymorphism? Malacologia 48 (1-2): 143-157.

Wilke T., Haase M., Hershler R., Liu H.-P., Misof B. \& Ponder W. 2013. Pushing short DNA fragments to the limit: phylogenetic relationships of 'hydrobioid' gastropods (Caenogastropoda: Rissooidea). Molecular Phylogenetics and Evolution 66 (3): 715-736. https://doi.org/10.1016/j.ympev.2012.10.025

Woodward J. 2009. The Physical Geography of the Mediterranean. OUP Oxford.

Manuscript received: 22 October 2019

Manuscript accepted: 7 April 2020

Published on: 19 May 2020

Topic editor: Rudy Jocqué

Section editor: Thierry Backeljau

Desk editor: Pepe Fernández 
KHALLOUFI N. et al., New hydrobiid genera and species from Tunisia

Printed versions of all papers are also deposited in the libraries of the institutes that are members of the EJT consortium: Muséum national d'histoire naturelle, Paris, France; Meise Botanic Garden, Belgium; Royal Museum for Central Africa, Tervuren, Belgium; Royal Belgian Institute of Natural Sciences, Brussels, Belgium; Natural History Museum of Denmark, Copenhagen, Denmark; Naturalis Biodiversity Center, Leiden, the Netherlands; Museo Nacional de Ciencias Naturales-CSIC, Madrid, Spain; Real Jardín Botánico de Madrid CSIC, Spain; Zoological Research Museum Alexander Koenig, Bonn, Germany; National Museum, Prague, Czech Republic. 